\title{
FINITE ELEMENT ANALYSIS OF NON-NEWTONIAN MAGNETO-HEMODYNAMIC FLOW CONVEYING NANO- PARTICLES THROUGH A STENOSED CORONARY ARTERY
}

\author{
B. VASU ${ }^{\mathrm{a}, *}$, ANKITA DUBEY ${ }^{\mathrm{a}}$ and O. ANWAR BÉG ${ }^{\mathrm{b}}$ \\ ${ }^{a}$ Department of Mathematics, Motilal Nehru National Institute of Technology Allahabad, Prayagraj, \\ Uttar Pradesh-211004, India \\ *Corresponding author-email: bvasu@mnnit.ac.in, rma1601@mnnit.ac.in \\ ${ }^{b}$ Department of Mechanical and Aeronautical Engineering, Salford University, Manchester, M54WT, \\ UK.‥A.Beg@salford.ac.uk
}

\begin{abstract}
The present study considers two-dimensional mathematical modelling of non-Newtonian nanofluid hemodynamics with heat and mass transfer in a stenosed coronary artery in the presence of a radial magnetic field. The second-grade differential viscoelastic constitutive model is adopted for blood to mimic non-Newtonian characteristics and blood is considered to contain a homogenous suspension of nanoparticles. Vogel's model is employed to simulate the variation of blood viscosity as a function of temperature. The governing equations are an extension of the Navier-Stokes equations with linear Boussinesq's approximation and Buongiorno's nanoscale model (which simulates both heat and mass transfer). The conservation equations are normalized by employing appropriate non-dimensional variables. It is assumed that the maximum height of the stenosis is small in comparison with the radius of the artery and furthermore that the radius of the artery and length of the stenotic region are of comparable magnitude. To study the influence of vessel geometry on blood flow and nano-particle transport, variation in the design and size of the stenosis is considered in the domain. The transformed equations are solved numerically by means of the finite element method based on the variational approach and simulated using the FreeFEM++ code. A detailed grid-independence study is included. Blood flow, heat and mass transfer characteristics are examined for the effects of selected geometric, nanoscale, rheological, viscosity and magnetic parameters i.e. stenotic diameter $(d)$, viscoelastic parameter $\left(\lambda_{1}\right)$, thermophoresis parameter $\left(N_{t}\right)$, Brownian motion parameter $\left(N_{b}\right)$ and magnetic body force parameter $(M)$ at the throat of the stenosis and throughout the arterial domain. The velocity, temperature and nanoparticle concentration fields are also visualized through instantaneous patterns of contours. An increase in magnetic and thermophoresis parameters is found to enhance the temperature, nanoparticle concentration and skin-friction coefficient. Increasing Brownian motion parameter is observed to accelerate the blood flow. Narrower stenosis significantly alters the temperature and nano-particle distributions and magnitudes. The novelty of the study relates to the combination of geometric complexity, multi-physical nanoscale and thermomagnetic behaviour and also the simultaneous presence of bio-rheological behaviour (all of which arise in actual cardiovascular heat transfer phenomena) in a single work with extensive visualization of the flow, heat and mass transfer characteristics. The simulations are relevant to diffusion of nanodrugs in magnetic targeted treatment of stenosed arterial disease.
\end{abstract}

KEYWORDS: Arterial stenosis, Non-Newtonian blood flow, Nano-drugs, Vogel's model, Magnetohydrodynamics, Thermophoresis, Finite Element Method. 


\section{NOMENCLATURE}

$A, B \quad$ Vogel's viscosity parameters

$A_{1}, A_{2} \quad$ Rivlin-Ericksen tensors

$B_{0} \quad$ Magnetic field

$B_{\mathrm{r}} \quad$ Brownian diffusion constant

$B^{\prime} \quad$ Total magnetic field

$D_{b} \quad$ Brownian diffusion coefficient

$D_{T} \quad$ Thermophoretic diffusion coefficient

g Gravitational vector

$J \quad$ Electric current density

$G_{r} \quad$ Grashof Number

$M \quad$ Magnetohydrodynamic body force parameter

$N_{b} \quad$ Brownian motion parameter

\section{Greek letters}

$\begin{array}{cl}\alpha_{1}, \alpha_{2} & \text { Material modules } \\ \kappa & \text { Thermal conductivity } \\ \rho_{f} & \text { Density of the base fluid } \\ \lambda_{1} & \text { Viscoelastic parameter } \\ \mu & \text { Dynamic viscosity } \\ \theta_{w} & \text { Wall Temperature } \\ \phi_{w} & \text { Concentration at wall } \\ \delta & \text { Stenosis depth } \\ \delta^{*} & \text { Ratio of stenosis depth to non-stenotic radius }\end{array}$
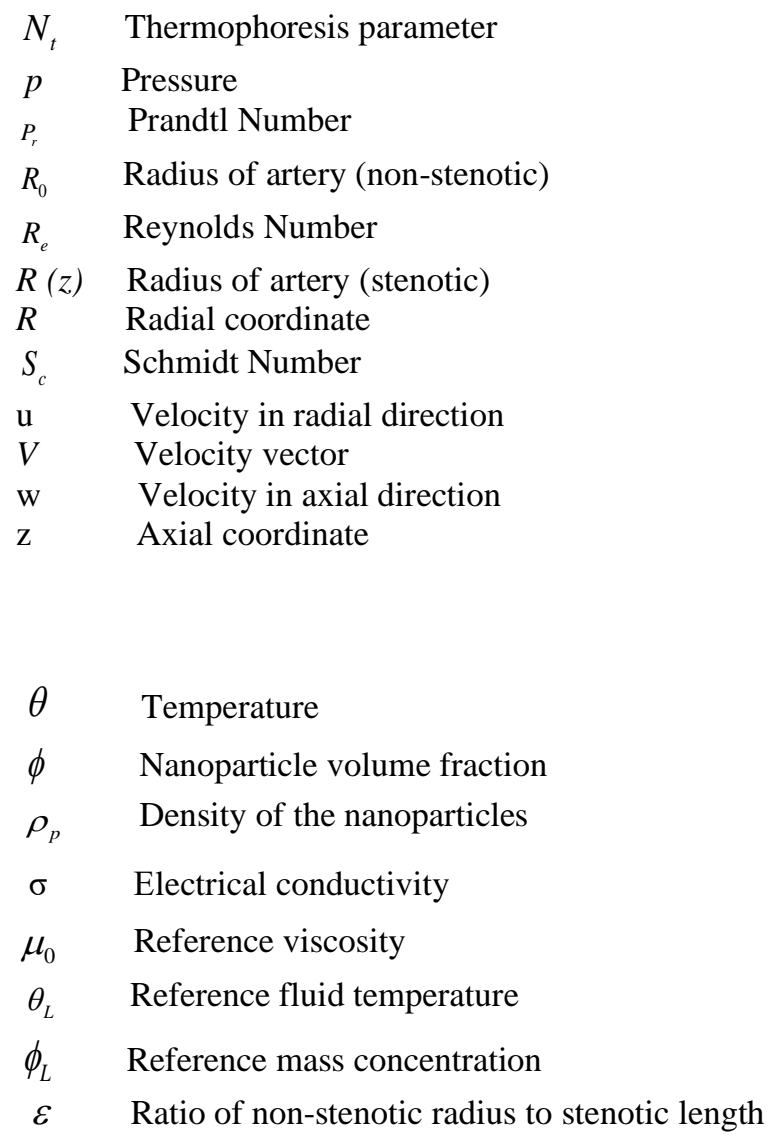

\section{INTRODUCTION}

Hemodynamics plays a critical role in the formation and evolution of cardiovascular diseases. Simulation of blood flow has been widely used in recent decades for better understanding the symptomatic spectrum of various diseases, in order to improve already existing treatments or to develop new therapeutic techniques. The characteristics of the blood flow in an artery can be modified significantly by arterial disease which may include aneurysms and stenoses [1]. The progress of atherosclerosis or stenosis in a blood vessel is quite common and may be accelerated by continuous addition of lipids in the arterial wall. Blood vessels are tapered by the growth of atherosclerotic plaques that bulge into the lumen, resulting in stenosed blood vessels. When stenosis is developed in an artery, one of the most severe problems associated with this obstruction is the increased resistance and the concomitant reduction of the blood flow to the specific vascular bed supplied by the artery. Thus, the development of a stenosis can lead to severe circulatory and arterial disorders [2].

Blood is suspension of various tiny particles in a continuous saline plasma solution. The plasma generally behaves as a Newtonian fluid whereas the whole blood (a suspension of cells and highly 
viscous in nature), exhibits the property of a non-Newtonian fluid, in particular in smaller vessels [3]. The non-Newtonian characteristics of blood emerge at low shear rate whereas at high shear rate blood usually behaves as a Newtonian fluid as observed in large arteries $[4,5]$. Blood flow ensures the transportation of nutrients, hormones, metabolic wastes, $\mathrm{O}_{2}$ and $\mathrm{CO}_{2}$ throughout the body to maintain cell-level metabolism, the regulation of the $\mathrm{pH}$, osmotic pressure and temperature of the whole body and protection from microbial and mechanical harms. The presence of arterial stenosis influence the hemodynamic behaviour of blood flow [6]. Arterial blood flow is fundamental the human circulatory system. Interest in this area has made important contributions to combatting heart diseases (stenosis) via diagnostic tools and simulations of chirurgical treatments like stents and by-passes [7]. Mekheimer and El kot [8] have analyzed blood flow in time-variant stenosed elastic tapered arteries. Riahi et al. [9] have examined blood flow in an artery containing an overlapping stenosis. Ellahi et al. [10] discussed the arterial blood flow through a mild composite stenosis by treating blood as micropolar fluid. Tzirakis et al. [11] studied numerically non-Newtonian bio-magnetic fluid flow in a duct. Akbar et al. [12] employed the Jeffrey fluid model for blood flow through a tapered artery with a stenosis to study non-Newtonian fluid characteristics in which a convective derivative rather than a time derivative was employed to simulate relaxation and retardation times. Chakravarty et al. [13] developed a mathematical model of blood flow through the time-variant tapered stenotic artery. Moreno and Kiran [14] explored plaque morphology in stenotic blood flows. Haghighi and Chalak [15] used a finite difference method and the Sisko rheological model to simulate blood flow in a stenotic artery with body acceleration effects. Tripathi [16] presented analytical solutions for three layered oscillatory blood flow via stenosed arteries. Mishra et al. [17] studied the blood flow through a composite stenosis in an artery with a permeable wall. Nadeem and Akbar [18] used a perturbation method and second grade viscoelastic model to investigate blood flow through a tapered artery. Ramesh and Devakar [19] have considered endoscopic and heat transfer effects in peristaltic nonNewtonian blood flow in a tube. Srivastav [20] explored permeability effects on the flow characteristics of a Newtonian fluid in an inserted catheterized stenosed artery. Jayaraman and Tewari [21] discussed the blood flow analysis in a catheterized curved artery by considering the blood vessel as a curved pipe and the catheter to be coaxial to it. Srivastava and Srivastava [22] presented a theoretical analysis on hemodynamics with artery catheterization with and without stenosis.

Magnetohydrodynamics (MHD) involves the motion of electrically-conducting fluids under the influence of an applied magnetic field. MHD arises in both Newtonian and non-Newtonian fluid 
flows and has diverse technological applications including medical engineering, chemical engineering, energy systems, materials processing etc. The presence of ions and iron in the haemoglobin molecule produces electrically-conducting properties in blood. Streaming blood can therefore be manipulated via the application of extra-corporeal magnetic fields which may be static or alternating in nature. Arterial diseases such as arteriosclerosis may therefore be treated via biomagnetic therapy. Magnetohydrodynamic blood flows also feature in electromagnetic medical pumps wherein magnetic field can be used to regulate the flow rates in the blood pump specific to different cardiac operations. In diseased arteries, the effect of vessels tapering in addition to the shape of a stenosis also constitutes an interesting scenario for magnetic blood flow simulation. Haik et al. [23] reported a 30\% decrease in blood flow rate due to a high magnetic field of 10 Tesla when gravitational effects are considered in non-stenosed flows. Yadav et al. [24] showed a similar reduction in blood flow rate but at a much smaller magnetic field of $0.002 \mathrm{~T}$ for stenosed arteries. Nadeem et al. [25] discussed the effects of induced magnetic field on blood flow through stenosed vessels. These studies have shown that the imposition of a magnetic field to streaming blood induces both electric and magnetic fields which interact to generate a Lorentzian body force, which is resistive in nature and opposes the movement of blood [26, 27]. A non-invasive technique based on MRI devices is often used to identify the structure of the stenosis in the artery, and this technique employs a strong magnetic field which affects the velocity field [28]. Many different mathematical and computational studies have been reported on the influence of magnetization in arterial blood flow. Tzirtzilakis [29] presented a detailed mathematical model for threedimensional biomagnetic blood flow under static magnetic field which featured ferrohydrodynamic and a Langevin function. They obtained extensive numerical results with a finite-difference-based pressure-linked collocated pseudo-transient method. Selvi and Ponalagusamy [30] investigated the effect of magnetic field on the two-phase oscillatory blood flow by assuming core and plasma regions as a Newtonian fluid in the arterial stenosis, showing that an increment in magnetic field elevates flow resistance of the blood flow in the stenosed artery. Ponalagusamy and Priyadharshini [31] extended the study [30] to consider tapered stenotic and non-Newtonian effects in magnetized oscillatory two-phase blood flow.

In recent years another significant development in biomedical engineering has been the application of nanofluids. The term "nanofluid" was first proposed about a decade ago by Choi [32], to characterize engineered colloids composed of nanoparticles dispersed in a base fluid. In comparison with milli- and micro- sized particle slurries explored in past, the nanoparticles are closer in molecular dimension to the particles of the base fluid. The popular base fluids are oil, 
water and organic fluids such as ethylene glycol and ethanol. Buongiorno [33] presented a comprehensive study of convective transport in nanofluids. Nanofluids display an enhancement in thermal conductivity, which is boosted with increasing volumetric fraction of nanoparticles as reported by Masuda et al. [34]. Nanoparticle doping has been implemented in many diverse technological sectors including heat exchangers, cooling of electronics, diesel electric generators, drug delivery and biomedical therapy. Classical models are not applicable for the enhancement of thermal conductivity of nanofluid. In recent years, most mathematical models have featured one or two postulated mechanisms for nanofluid heat transfer, irrespective of the area of application. As a result, there is relatively sparse fundamental research which has been reported on determination of the effective thermal diffusivity or heat transfer coefficients for nanofluids in natural convection, as highlighted by Wong et al. [35]. However Xuan and Roetzel [36] and Xuan and $\mathrm{Li}$ [37] assumed that convective heat transfer enhancement is due mainly to dispersion of the suspended nanoparticles. Giljohann et al. [38] and Kumar et al. [39] also investigated the effect of gold nanoparticles suspension in blood identifying the excellent utility of small size nanoparticles in biomedical systems. In recent years, various nano-sized particles have been deployed in pharmacodynamics to increase or decrease the blood capillary growth and most of them are very effective. Nano-particles have also been employed in biological fuel cell systems. Bég et al. [40] studied combined nanofluid doping and oxytactic biconvection micro-organisms in near-wall flows of microbial fuel cells. Ali et al. [41] presented a comprehensive computational model for studied unsteady heat and mass transfer in streaming blood flow doped with nanoparticles via a tapered stenotic artery.

In many hemodynamic simulations, blood viscosity is assumed to be constant. However, this assumption is not valid everywhere. In general, the coefficient of viscosity for real fluids is a function of temperature and pressure. In recent years there have been several investigations in which blood viscosity has been taken as function of temperature. Vogel's model is commonly used to describe the variation of the viscosity with temperature in a variety of flows [42]. Pakdemirlia and Yilbas [43] utilized the Vogel viscosity model to analyse entropy generation in viscoelastic blood flow. Ellahi et al. [44] derived homotopy series solutions for non-Newtonian nanofluid flows using two variable viscosity models (Reynolds' model and Vogel's model). Akbar et al. [45] employed Vogel's model to consider viscosity variation in peristaltic flow of Jeffrey fluid. Malik et al. [46] investigated variable viscosity in Eyring-Powell flows. Hatami et al. [47] simulated hydromagnetic convection in axisymmetric viscoelastic flow doped with gold nano-particles. They 
observed that non-Newtonian and viscosity parameters had a significant influence on the temperature and velocity profiles in physiological flows.

The theoretical studies dealing with effects of heat transfer and magnetic field on the steady flow of blood in a coronary artery have received comparatively less attention. The vast majority of models in the literature relating to thermomagnetic blood flows have considered very simplified geometric systems and have not presented beyond one-dimensional formulations. It has been observed that the heart rate decreases by exposing biological systems to an external magnetic field and also that injected nanoparticles help in improving the blood flow in a stenosed artery. Motivated by extending these studies this paper focuses on simulation of the nanoparticle drug delivery in stenosed realistic hemodynamics in small vessels (coronary arteries) of the human circulatory system where blood is considered as second grade fluid with flow viscosity variation simulated as a function of temperature which is adequately enabled in the Vogel model. The results elaborate on the influence of several nanoscale parameters (thermophoresis parameter and Brownian motion parameter) and magnetic body force parameter on velocity, skin friction coefficient, nanoparticle concentration, temperature profiles and wall shear stress on the stenotic section in addition to the rest of the coronary artery. Due to the viscosity being modelled as a function of temperature, an improved prediction of velocity and wall shear stress has been achieved which approximates more closely to actual blood flow in stenotic artery. A small segment of stenosed coronary artery is considered in which the non-Newtonian blood flow and heat transfer with nano particles is modelled by employing the Buongiorno model. The normalized conservation equations are solved subject to appropriate boundary conditions with the finite element method using a variational approach and the FreeFem++ code. Overall the novelty of the study may be summarized in that it provides a significant step forward in combining the geometric complexity, multi-physical nanoscale thermomagnetic behaviour and the interaction of many real phenomena (rheological second order effects etc) in biological cardiovascular heat transfer simulations in a single work with extensive visualization of the flow, heat and mass transfer characteristics. The present simulations are envisaged to be of relevance to better characterizing hemodynamics in the assessment and diagnosis of cardiovascular disease, testing the hypothesis of disease formation, modelling the transport of drugs through the circulatory systems and furthermore may be of benefit in the design of cardiovascular devices, heart valves, stents, probes etc.

\section{MAGNETIC NON-NEWTONIAN NANO-PARTICLE BLOOD FLOW MODEL}


A two-dimensional mathematical model for blood flow in a coronary artery is considered wherein blood flow is modelled as non-homogeneous fluid flow containing a suspension of nanoparticles. Blood rheology is simulated with the second grade Reiner-Rivlin fluid model. Both heat and mass transfer are included. For the simulation, the stationary case of the blood vessel is assumed i.e. the pulsatile nature of streaming blood is neglected. The velocity is taken as zero at the internal walls of the vessel which is modelled as a cylindrical tube. A cylindrical coordinate $\operatorname{system}(r, \varphi, z)$ is therefore adopted, where $r$ is the radial coordinate, $z$ - is axial coordinate and $\varphi$ is azimuthal coordinate. Since the flow is axisymmetric the contribution in the azimuthal $(\varphi)$ direction may be neglected i.e. the flow is only in the radial $(r)$ and axial $(z)$ direction as shown in Fig. (1). The fluid is incompressible and laminar. The coronary artery has finite length $L$ and contains a sinusoidalshaped stenosis. In the arterial segment the geometry of the mild stenosis is assumed to be is

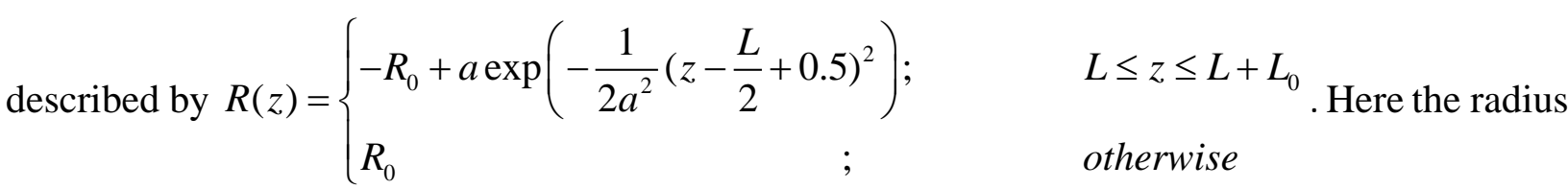
and length of the non-stenotic section are $R_{0}, L_{0}$ respectively and the radius of the stenotic part is taken as $R(z)$. The $z$-axis is parallel to the blood flow direction and normal to the $r$-axis and $d$ is the stenotic diameter. The obstruction (stenosis) is symmetrical with a maximum height $\delta$ with $a=\frac{5}{4} \delta$.

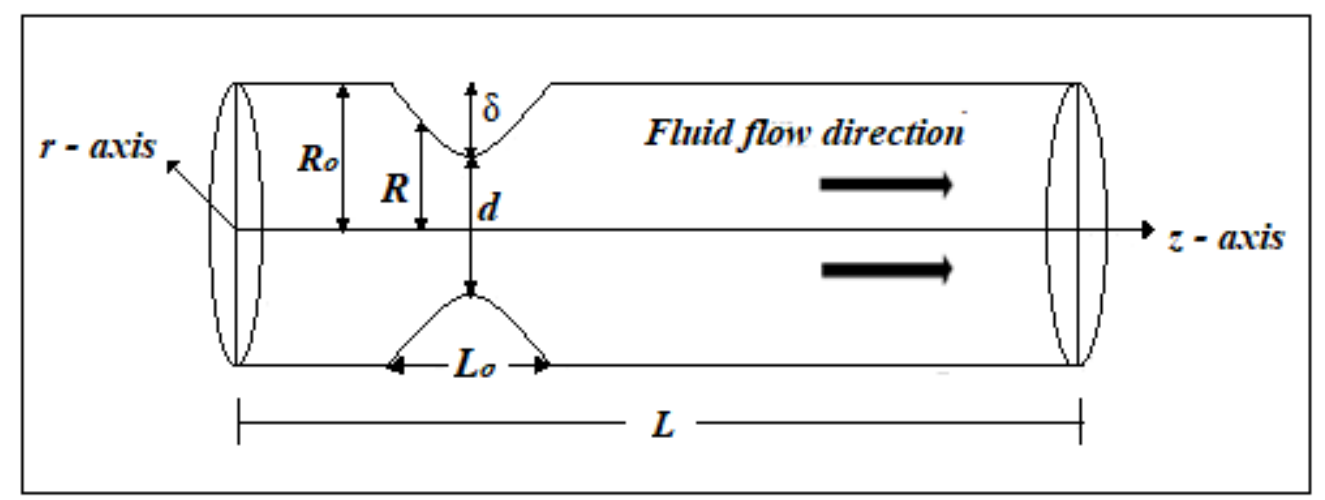

Figure 1. Schematic illustration of a stenosed coronary artery

For steady axisymmetric flow of blood in the arterial vessel, the velocity vector $V$ is assumed to be of the form:

$$
V=[u(r, z), 0, w(r, z)]
$$


Here $u$ and $w$ are the velocity components in radial and axial directions. Blood is considered to be an incompressible second-grade non-Newtonian fluid containing a homogenous distribution of nano-particles. The viscosity of blood is assumed to be a function of temperature following Vogel's model $[42,44]$ :

$$
\mu=\mu_{0} e^{\frac{A}{B+\theta}-\theta_{L}}
$$

Since natural convection is considered, the appropriate expression for the nanofluid density [33, 49] is:

$$
\begin{aligned}
& \rho=\phi \rho_{p}+(1-\phi) \rho_{f_{0}} \\
& \cong \phi \rho_{p}+(1-\phi)\left[\rho_{f}\left(1-\beta_{T}\left(\theta-\theta_{L}\right)\right)\right]
\end{aligned}
$$

Here $\rho_{f_{0}}$ is base fluid (blood) density and $\rho_{f}$ is the base fluid's density at the reference fluid temperature $\theta_{L}$.

The conservation equations for mass, momentum, thermal energy and nanoparticle volume fraction (species) for the nano-doped blood transport may be presented in vectorial form as:

$$
\begin{gathered}
\nabla \cdot V(r, z)=0 \\
\rho_{f}\left(\frac{\partial V}{\partial t}+(V \cdot \nabla) V\right)=\operatorname{div} T_{s}+\left[\phi \rho_{p}+(1-\phi)\left[\rho_{f}\left(1-\beta_{T}\left(\theta-\theta_{L}\right)\right)\right]\right] g+J \times B^{\prime} \\
(\rho c)_{f}\left(\frac{\partial \theta}{\partial t}+(V \cdot \nabla) \theta\right)=k \nabla^{2} \theta+(\rho c)_{p}\left[D_{b}(\nabla \phi \cdot \nabla \theta)+\frac{D_{T}}{\theta_{L}}(\nabla \theta \cdot \nabla \theta)\right] \\
\left(\frac{\partial \phi}{\partial t}+(V \cdot \nabla) \phi\right)=D_{b} \nabla^{2} \phi+\frac{D_{T}}{\theta_{L}} \nabla^{2} \theta
\end{gathered}
$$

Here $\phi$ is the nanoparticle volume fraction. In equation (5) the third term on the right-hand side is the contribution due to applied magnetic field in electrically-conducting blood flow. Ohm's law provides a relation between $J$ and $B^{\prime}[50]$ :

$$
J=\sigma\left(E+V \times B^{\prime}\right)
$$

Where $E$ represents the electric field, $B^{\prime}=B_{0}+b$ represents the total magnetic field, $\sigma$ the electrical conductivity, $\boldsymbol{V}$ is the velocity vector and $\boldsymbol{J}$ represents the electric current density. For small magnetic Reynolds number, the induced magnetic field is neglected. Hence:

$$
J \times B^{\prime}=-\sigma B_{0}{ }^{2} V
$$

In this study, it is assumed that each variable, $u, v, \theta$ and $\phi$ has an initial value at the boundary wall and this value is prescribed as unity at the inlet boundary and zero at the outlet. 
The constitutive equation for the Cauchy stress tensor in a second-order Reiner- Rivlin fluid is given by [51]

$$
T_{S}=-p I+\mu A_{1}+\alpha_{1} A_{2}+\alpha_{2} A_{1}^{2}
$$

Where $\alpha_{1}, \alpha_{2}$ are material moduli which in general are considered to be functions of temperature.

In Eq. (10), due to the restraint of incompressibility, $-p I$ represents the spherical stress, and the kinematical tensors $A_{1}, A_{2}$ can be defined by the following equations:

$$
\begin{gathered}
A_{1}=\nabla V+(\nabla V)^{t} \\
A_{n}=\frac{d A_{n-1}}{d t}+A_{n-1}(\nabla V)+(\nabla V)^{t} A_{n-1}
\end{gathered}
$$

For the model to be compatible with thermodynamics, in the sense that all motions satisfy the Clausius-Duhelm inequality, it is assumed that the specific Helmholtz free energy is minimum in equilibrium. Therefore, all the material derivatives taken in the above equations must meet the following conditions (Dunn and Fosdick [52] and Dunn and Rajagopal [53]).

$$
\mu \geq 0, \alpha_{1} \geq 0 \text { and } \quad \alpha_{1}+\alpha_{2}=0
$$

From the above Eqns. (4) - (7), neglecting the pressure gradient, the steady state, incompressible, two-dimensional governing equations for the nano-doped hydromagnetic blood transport reduce to the following:

$$
\begin{gathered}
\frac{\partial u}{\partial r}+\frac{u}{r}+\frac{\partial w}{\partial z}=0 \\
\frac{\partial p}{\partial r}=\mu\left[\frac{\partial^{2} w}{\partial z \partial r}-\frac{2 u}{r^{2}}\right]+\frac{\partial \mu}{\partial z} \frac{\partial w}{\partial r}+\alpha_{1}\left(-\frac{1}{r}\left(\frac{\partial w}{\partial r}\right)^{2}+2 \frac{\partial w}{\partial r} \frac{\partial^{2} w}{\partial r^{2}}+2 \frac{\partial w}{\partial z} \frac{\partial^{2} w}{\partial r \partial z}+\frac{\partial w}{\partial r} \frac{\partial^{2} w}{\partial z^{2}}\right. \\
\left.+u \frac{\partial^{3} w}{\partial z \partial r^{2}}+w \frac{\partial^{3} w}{\partial z^{2} \partial r}+\frac{\partial w}{\partial z} \frac{\partial^{2} w}{\partial r \partial z}+2 \frac{u^{2}}{r^{4}}\right)-\left(\phi \rho_{p}+(1-\phi)\left[\rho_{f}\left(1-\beta_{T}\left(\theta-\theta_{L}\right)\right)\right]\right) g-\sigma B_{0}{ }^{2} u \\
\rho_{f}\left(u \frac{\partial w}{\partial r}+w \frac{\partial w}{\partial z}\right)=-\frac{\partial p}{\partial z}+\mu\left(\frac{\partial^{2} w}{\partial r^{2}}+\frac{1}{r} \frac{\partial w}{\partial r}\right)+\frac{\partial \mu}{\partial r} \frac{\partial w}{\partial r}+\alpha_{1}\left[u \frac{\partial^{3} w}{\partial r^{3}}-\frac{\partial w}{\partial r} \frac{\partial^{2} u}{\partial r^{2}}\right. \\
+\frac{\partial w}{\partial z} \frac{\partial^{2} w}{\partial r^{2}}+w \frac{\partial^{3} w}{\partial r^{2} \partial z}+\frac{u}{r} \frac{\partial^{2} w}{\partial r^{2}}+\frac{w}{r} \frac{\partial^{2} w}{\partial r \partial z}+\frac{1}{r} \frac{\partial w}{\partial r} \frac{\partial w}{\partial z}-\frac{1}{r} \frac{\partial u}{\partial r} \frac{\partial w}{\partial r} \\
\left.+\frac{\partial w}{\partial r} \frac{\partial^{2} w}{\partial r \partial z}+2 \frac{\partial w}{\partial r} \frac{\partial^{2} w}{\partial z \partial r}+2 u \frac{\partial^{3} w}{\partial r \partial z^{2}}+2 w \frac{\partial^{3} w}{\partial z^{3}}+2 \frac{\partial w}{\partial z} \frac{\partial^{2} w}{\partial z^{2}}\right] \\
+\left(\phi \rho_{p}+(1-\phi)\left[\rho_{f}\left(1-\beta_{T}\left(\theta-\theta_{L}\right)\right)\right]\right) g-\sigma B_{0}{ }^{2} w
\end{gathered}
$$




$$
\begin{gathered}
(c \rho)_{f}\left(u \frac{\partial \theta}{\partial r}+w \frac{\partial \theta}{\partial z}\right)=\kappa\left(\frac{\partial^{2} \theta}{\partial r^{2}}+\frac{1}{r} \frac{\partial \theta}{\partial r}+\frac{\partial^{2} \theta}{\partial z^{2}}\right)+(c \rho)_{p}\left(D_{b}\left[\frac{\partial \phi}{\partial r} \frac{\partial \theta}{\partial r}+\frac{\partial \phi}{\partial z} \frac{\partial \theta}{\partial z}\right]\right. \\
\left.+\frac{D_{T}}{\theta_{L}}\left[\frac{\partial \theta}{\partial r} \frac{\partial \theta}{\partial r}+\frac{\partial \theta}{\partial z} \frac{\partial \theta}{\partial z}\right]\right) \\
\left(u \frac{\partial \phi}{\partial r}+w \frac{\partial \phi}{\partial z}\right)=D_{b}\left(\frac{\partial^{2} \phi}{\partial r^{2}}+\frac{1}{r} \frac{\partial \phi}{\partial r}+\frac{\partial^{2} \phi}{\partial z^{2}}\right)+\frac{D_{T}}{\theta_{L}}\left(\frac{\partial^{2} \theta}{\partial r^{2}}+\frac{1}{r} \frac{\partial \theta}{\partial r}+\frac{\partial^{2} \theta}{\partial z^{2}}\right)
\end{gathered}
$$

The corresponding boundary conditions are:

$$
\begin{array}{ll}
u=0, w=U_{0}, \frac{\partial w}{\partial r}=0, \frac{\partial \theta}{\partial r}=0, \frac{\partial \phi}{\partial r}=0 & \text { at } r=0 \\
u=0, w=0, \theta=\theta_{w}, \phi=\phi_{w} & \text { at } r=R(z)
\end{array}
$$

The following non-dimensional parameters are introduced:

$$
\begin{aligned}
& \bar{u}=\frac{u L_{0}}{U_{0} \delta}, \bar{w}=\frac{w}{U_{0}}, \bar{z}=\frac{z}{L_{0}}, \bar{r}=\frac{r}{R_{0}}, \bar{\mu}=\frac{\mu}{\mu_{0}} \\
& \bar{p}=\frac{p R_{0}^{2}}{U_{0} \mu_{0} L_{0}}, \quad \bar{\theta}=\frac{\theta-\theta_{L}}{\theta_{w}-\theta_{L}} \quad \text { and } \quad \bar{\phi}=\frac{\phi-\phi_{L}}{\phi_{w}-\phi_{L}}
\end{aligned}
$$

Where $U_{0}, L_{0}, R_{0}, \mu_{0}, \delta, \theta_{L}, \theta_{w}, \phi_{L}$ and $\phi_{w}$ denote the reference velocity, reference length of the blood vessel, reference radius, reference dynamic viscosity, stenosis depth, reference fluid temperature, vessel wall temperature, reference mass concentration and vessel wall mass concentration in the arterial tube model, respectively.

The two important physical quantities of interest are the wall shear rate and skin friction.

The shear rate at wall is

$$
\tau_{w}=-\left(\mu \frac{\partial w}{\partial r}+\alpha_{1}\left(u \frac{\partial^{2} w}{\partial r^{2}}+w \frac{\partial^{2} w}{\partial r \partial z}-\frac{\partial w}{\partial r} \frac{\partial w}{\partial z}+\frac{\partial u}{\partial r} \frac{\partial w}{\partial r}\right)\right)
$$

Skin-friction is defined as follows:

$$
C_{f}=\frac{2 \tau_{w}}{\rho_{f} U_{0}^{2}}
$$

Implementing Eqns. (20) in eqns. (14)-(19) the following system of dimensionless conservation equations emerges: 


$$
\begin{aligned}
& \varepsilon^{2} \frac{\partial \bar{p}}{\partial \bar{r}}=\bar{\mu}\left[\varepsilon \frac{\partial^{2} \bar{w}}{\partial \bar{z} \partial \bar{r}}-\delta^{*} \varepsilon \frac{2 \bar{u}}{\bar{r}^{2}}\right]+\frac{\partial \bar{\mu}}{\partial \bar{z}} \frac{\partial \bar{w}}{\partial \bar{r}}+\lambda_{1}\left[\frac{1}{\bar{r}}\left(\frac{\partial \bar{w}}{\partial \bar{r}}\right)^{2}+2 \varepsilon^{2} \frac{\partial \bar{w}}{\partial \bar{z}} \frac{\partial^{2} \bar{w}}{\partial \bar{r} \partial \bar{z}}+2 \frac{\partial \bar{w}}{\partial \bar{r}} \frac{\partial^{2} \bar{w}}{\partial \bar{r}^{2}}+\varepsilon^{2} \frac{\partial \bar{w}}{\partial \bar{r}} \frac{\partial^{2} \bar{w}}{\partial \bar{z}^{2}}\right. \\
& \left.+\delta^{*} \varepsilon^{2} u \frac{\partial^{3} \bar{w}}{\partial \bar{z} \partial \bar{r}^{2}}-\delta^{*} \varepsilon^{2} \frac{\partial \bar{u}}{\partial \bar{r}} \frac{\partial^{2} \bar{w}}{\partial \bar{r} \partial \bar{z}}+\varepsilon^{2} w \frac{\partial^{3} \bar{w}}{\partial \bar{z}^{2} \partial \bar{r}}+2\left(\delta^{*}\right)^{2} \varepsilon \frac{\bar{u}^{2}}{\bar{r}^{4}}\right]+\left(B_{r} \bar{\phi}+G_{r} \bar{\theta}\right)-\delta^{*} \varepsilon M \bar{u} \\
& \operatorname{Re}\left(\delta^{*} \varepsilon \bar{u} \frac{\partial \bar{w}}{\partial \bar{r}}+\varepsilon \bar{w} \frac{\partial \bar{w}}{\partial \bar{z}}\right)=-\varepsilon^{3} \frac{\partial \bar{p}}{\partial \bar{z}}+\bar{\mu}\left(\varepsilon \frac{\partial^{2} \bar{w}}{\partial \bar{r}^{2}}+\varepsilon \frac{1}{\bar{r}} \frac{\partial \bar{w}}{\partial \bar{r}}\right)+\frac{\partial \bar{\mu}}{\partial \bar{r}} \frac{\partial \bar{w}}{\partial \bar{r}}+\lambda_{1}\left(\delta^{*} \bar{u} \frac{\partial^{3} \bar{w}}{\partial \bar{r}^{3}}-\delta^{*} \varepsilon \frac{\partial \bar{u}}{\partial \bar{r}} \frac{\partial \bar{w}}{\partial \bar{r}}\right. \\
& +\frac{\partial \bar{w}}{\partial \bar{z}} \frac{\partial^{2} \bar{w}}{\partial r^{2}}+\bar{w} \frac{\partial^{3} \bar{w}}{\partial \bar{r}^{2} \partial \bar{z}}+\delta^{*} \frac{\bar{u}}{\bar{r}} \frac{\partial^{2} \bar{w}}{\partial \bar{r}^{2}}+\frac{\bar{w}}{\bar{r}} \frac{\partial^{2} \bar{w}}{\partial \bar{r} \partial \bar{z}}+\frac{1}{\bar{r}} \frac{\partial \bar{w}}{\partial \bar{r}} \frac{\partial \bar{w}}{\partial \bar{z}}+\frac{\partial \bar{w}}{\partial \bar{r}} \frac{\partial^{2} \bar{w}}{\partial \bar{r} \partial \bar{z}}-\delta^{*} \varepsilon \frac{\partial \bar{w}}{\partial r} \frac{\partial^{2} \bar{u}}{\partial r^{2}} \\
& \left.+2 \frac{\partial \bar{w}}{\partial \bar{r}} \frac{\partial^{2} \bar{w}}{\partial \bar{z} \partial \bar{r}}+2 \delta^{*} \varepsilon^{2} \bar{u} \frac{\partial^{3} \bar{w}}{\partial \bar{r} \partial \bar{z}^{2}}+2 \varepsilon \bar{w} \frac{\partial^{3} \bar{w}}{\partial \bar{z}^{3}}+2 \varepsilon \frac{\partial \bar{w}}{\partial \bar{z}} \frac{\partial^{2} \bar{w}}{\partial \bar{z}^{2}}\right)+G_{r} \bar{\theta}+B_{r} \bar{\phi}-M \bar{w} \\
& \operatorname{Re} \operatorname{Pr}\left(\delta^{*} \varepsilon \bar{u} \frac{\partial \bar{\theta}}{\partial \bar{r}}+\varepsilon^{2} \bar{w} \frac{\partial \bar{\theta}}{\partial \bar{z}}\right)=\left(\frac{\partial^{2} \bar{\theta}}{\partial \bar{r}^{2}}+\frac{1}{\bar{r}} \frac{\partial \bar{\theta}}{\partial \bar{r}}+\varepsilon^{2} \frac{\partial^{2} \bar{\theta}}{\partial \bar{z}^{2}}\right)+N_{b}\left[\frac{\partial \bar{\phi}}{\partial \bar{r}} \frac{\partial \bar{\theta}}{\partial \bar{r}}+\varepsilon^{2} \frac{\partial \bar{\phi}}{\partial \bar{z}} \frac{\partial \bar{\theta}}{\partial \bar{z}}\right] \\
& +N_{t}\left[\frac{\partial \bar{\theta}}{\partial \bar{r}} \frac{\partial \bar{\theta}}{\partial \bar{r}}+\varepsilon^{2} \frac{\partial \bar{\theta}}{\partial \bar{z}} \frac{\partial \bar{\theta}}{\partial \bar{z}}\right] \\
& \operatorname{Re} L e\left(\delta^{*} \varepsilon^{2} \bar{u} \frac{\partial \bar{\phi}}{\partial \bar{r}}+\varepsilon^{2} \bar{w} \frac{\partial \bar{\phi}}{\partial \bar{z}}\right)=\left(\frac{\partial^{2} \bar{\phi}}{\partial \bar{r}^{2}}+\frac{1}{\bar{r}} \frac{\partial \bar{\phi}}{\partial \bar{r}}+\varepsilon^{2} \frac{\partial^{2} \bar{\phi}}{\partial \bar{z}^{2}}\right) \\
& +\frac{N_{t}}{N_{b}}\left(\frac{\partial^{2} \bar{\theta}}{\partial \bar{r}^{2}}+\frac{1}{\bar{r}} \frac{\partial \bar{\theta}}{\partial \bar{r}}+\varepsilon^{2} \frac{\partial^{2} \bar{\theta}}{\partial \bar{z}^{2}}\right)
\end{aligned}
$$

A mild stenosis is assumed and the nondimensional geometric parameters appearing in the terms defined above are stenosis height parameter $\left(\delta^{*}=\delta / R_{0}<<1\right)$ and the vessel aspect ratio $\left(\varepsilon=R_{0} / L_{0}\right.$ ). For the subsequent analysis, we shall assume that $\delta^{*} \ll 1$ and $\varepsilon=O$ (1), i.e., the maximum height of the stenosis is small in comparison with the radius of the artery and also that the radius of the artery and length of the stenotic region are of comparable magnitude. Hence these parameters should be neglected [54]. In this study viscosity of nanofluid is considered as a function of temperature as given in Vogel's model [46, 47, 48] we can rewrite Eqn. (2) as:

$$
\bar{\mu}=\frac{c}{S}\left[1-\frac{A \bar{\theta}}{B^{2}}\right], \quad \text { where } S=\mu_{0} e^{\left((A / B)-\theta_{L}\right)}
$$

Here $A, B, \mathrm{c}, \mu_{0}, \theta_{L}$ are the reference values.

After applying the above equation in the normalized system of Eqns. (23) - (26), we get:

$$
\frac{c A}{S B^{2}} \frac{\partial \bar{\theta}}{\partial \bar{z}} \frac{\partial \bar{w}}{\partial \bar{r}}-\lambda_{1}\left[\frac{1}{\bar{r}}\left(\frac{\partial \bar{w}}{\partial \bar{r}}\right)^{2}+2 \frac{\partial \bar{w}}{\partial \bar{r}} \frac{\partial^{2} \bar{w}}{\partial \bar{r}^{2}}\right]-\left(B_{r} \bar{\phi}+G_{r} \bar{\theta}\right)=0
$$




$$
\begin{gathered}
\frac{c A}{S B^{2}} \frac{\partial \bar{\theta}}{\partial \bar{r}} \frac{\partial \bar{w}}{\partial \bar{r}}-\lambda_{1}\left(\frac{\partial \bar{w}}{\partial \bar{z}} \frac{\partial^{2} \bar{w}}{\partial r^{2}}+\bar{w} \frac{\partial^{3} \bar{w}}{\partial \bar{r}^{2} \partial \bar{z}}+\frac{\bar{w}}{\bar{r}} \frac{\partial^{2} \bar{w}}{\partial \bar{r} \partial \bar{z}}+\frac{1}{\bar{r}} \frac{\partial \bar{w}}{\partial \bar{r}} \frac{\partial \bar{w}}{\partial \bar{z}}\right. \\
\left.+\frac{\partial \bar{w}}{\partial \bar{r}} \frac{\partial^{2} \bar{w}}{\partial \bar{r} \partial \bar{z}}+2 \frac{\partial \bar{w}}{\partial \bar{r}} \frac{\partial^{2} \bar{w}}{\partial \bar{z} \partial \bar{r}}\right)-\left(G_{r} \bar{\theta}+B_{r} \bar{\phi}\right)+M \bar{w}=0 \\
\left(\frac{\partial^{2} \bar{\theta}}{\partial \bar{r}^{2}}+\frac{1}{\bar{r}} \frac{\partial \bar{\theta}}{\partial \bar{r}}\right)+\left(N_{b}\left[\frac{\partial \bar{\phi}}{\partial \bar{r}} \frac{\partial \bar{\theta}}{\partial \bar{r}}\right]+N_{t}\left[\frac{\partial \bar{\theta}}{\partial \bar{r}} \frac{\partial \bar{\theta}}{\partial \bar{r}}\right]\right)=0 \\
\left(\frac{\partial^{2} \bar{\phi}}{\partial \bar{r}^{2}}+\frac{1}{\bar{r}} \frac{\partial \bar{\phi}}{\partial \bar{r}}\right)+\frac{N_{t}}{N_{b}}\left(\frac{\partial^{2} \bar{\theta}}{\partial \bar{r}^{2}}+\frac{1}{\bar{r}} \frac{\partial \bar{\theta}}{\partial \bar{r}}\right)=0
\end{gathered}
$$

The associated non-dimensional boundary conditions become:

$$
\begin{aligned}
& \bar{u}=0, \bar{w}=1, \frac{\partial \bar{w}}{\partial \bar{r}}=0, \frac{\partial \bar{\theta}}{\partial \bar{r}}=0, \frac{\partial \bar{\phi}}{\partial \bar{r}}=0 \quad \text { at } \bar{r}=0 \\
& \bar{u}=0, \bar{w}=0, \bar{\theta}=1, \bar{\phi}=1 \quad \text { at } \bar{r}=\bar{R}(z)
\end{aligned}
$$

In Eqns. (28)-(31) the following dimensionless quantities arise: MHD i.e. magnetic body force parameter $M=\frac{\sigma B_{0}{ }^{2} L_{0} R_{0}}{\mu_{0}}$, the Brownian motion parameter, $N_{b}=\frac{D_{b}\left(\phi_{w}-\phi_{L}\right)(\rho c)_{p}}{\kappa}$, thermophoresis parameter $N_{t}=\frac{D_{T}\left(\theta_{w}-\theta_{L}\right)(\rho c)_{p}}{\theta_{L} \kappa}$, the Grashof number $G r=\frac{\left(\theta_{w}-\theta_{L}\right) \rho_{f, w} g\left(1-\phi_{L}\right) L_{0} R_{0} \beta_{T}}{U_{0} \mu_{0}}$, the Brownian diffusion constant $B r=\frac{\left(\rho_{p}-\rho_{f}\right) L_{0} R_{0}\left(\phi_{w}-\phi_{L}\right) g}{U_{0} \mu_{0}}$, the Reynolds number $\operatorname{Re}=\frac{\rho_{f} U_{0} L_{0}}{\mu_{0}}$, viscoelastic parameter $\lambda_{1}=\frac{\alpha_{1} U_{0}}{\mu_{0} L_{0}}$, 14Prandtl number $\operatorname{Pr}=\frac{c_{p} \mu_{0}}{\kappa}$, and Schmidt number $S_{c}=\frac{\mu_{0}}{\rho_{f} D_{b}}$.

Non-dimensional skin friction coefficient is given by:

$$
\frac{1}{2} C_{f} \operatorname{Re}=\bar{\mu} \frac{\partial \bar{w}}{\partial \bar{r}}+\lambda_{1}\left(\bar{w} \frac{\partial^{2} \bar{w}}{\partial \bar{r} \partial \bar{z}}-\frac{\partial \bar{w}}{\partial \bar{r}} \frac{\partial \bar{w}}{\partial \bar{z}}\right)
$$

Applying equation (27), we get-

$$
\frac{1}{2} C_{f} \operatorname{Re}=\frac{c}{S}\left[1-\frac{A \bar{\theta}}{B^{2}}\right] \frac{\partial \bar{w}}{\partial \bar{r}}+\lambda_{1}\left(\bar{w} \frac{\partial^{2} \bar{w}}{\partial \bar{r} \partial \bar{z}}-\frac{\partial \bar{w}}{\partial \bar{r}} \frac{\partial \bar{w}}{\partial \bar{z}}\right)
$$

Where $R e$ is the local Reynolds number (which is the ratio of inertial to viscous forces).

\section{FINITE ELEMENT SIMULATION WITH FreeFEM++}

This section describes the numerical solution of the non-dimensional model derived in Sec. (2) i.e. the transformed Eqns. (28)-(31) with boundary conditions (32) using the finite element method with a variational approach and FreeFEM++ software. The finite element method involves dividing the domain of the problem into a collection of subdomains, with each subdomain represented by a set of element equations to the original problem, followed by systematically 
recombining all sets of element equations into a global system of equations for the final calculation. FEM's popularity has been increasing due to the greater flexibility it offers in modelling complex geometries. It has significant capabilities in accommodating general boundary conditions and variable material properties with a clear structure and versatility that helps to construct general purpose software for diverse applications. FEM has a solid theoretical foundation which gives added reliability and makes it possible to mathematically analyse and estimate the error in the approximate solution. Some recent studies employing the finite element method in blood flow modelling include Rajashekhar and Fabian [55] who investigated two-dimensional steady blood flow through an arterial bifurcation using finite element analysis (FEA) with different geometries. Many other studies of medical fluid dynamics have been reported using finite element techniques. Contreras-Silva et al. [56] conducted finite element simulations of blood flow in an ideal artery with stenosis. Lozovskiy et al. [57] developed a finite element method for incompressible viscous blood flow in a time-dependent domain using a quasi-Lagrangian formulation of the problem which provides stability and convergence analysis of the fully discrete (finite-difference in time and finite-element in space) method. Finite element modelling and simulation of the arterial network in the human arm for the aortic pulse wave propagation has been described by Choudhari et al. [58]. Gupta et al. [59] employed a finite element method to study drug diffusion in the human dermal region. They employed linear shape functions and discretized the dermal region into three layers by considering a linear concentration variation in each layer and defined as a function of one space variable. Kanday and Rafiq [60] applied the variational finite element method to study the absorption rate of drugs in a transdermal drug delivery system wherein the overall drug concentration was formulated by assembling the linear variations of each region. Jyoti and Srivastava [61] deployed a four-step wavelet Galerkin Method for parabolic and hyperbolic problems. Chi [62] used the discontinuous Galerkin method for time-dependent partial differential equations in medical transport problems.

FreeFEM++ is an open source multi-physics programming software which allows fast and efficient solution of partial differential equations using the finite element method. This software offers several triangular finite elements, including discontinuous elements. It also provides options for simulating coupled electromagnetics, heat and mass diffusion and other phenomena. Recently FreeFEM++ has been increasingly utilized by researchers in diverse fields of engineering science fluid dynamics. Bertoglio et al. [63] applied FreeFEM++ to simulate flow in a straight channel with space-time discretization and adaptive mesh refinement. Deleuze [64] used FreeFem++ to investigate the effect of an inserted needle on the subcutaneous interstitial flow to describe the physical stress affecting body cells during acupuncture. Further studies employing FreeFem++ 
software include Janela et al. [65] (on rigid particle motion in Newtonian fluids with a hyperviscosity model), Mahmud et al. [66] (on incompressible Newtonian fluid flow) and Devys et al. [67] (on two-dimensional respiratory hydrodynamics).

To obtain a weak formulation of the system of differential equations (19-23) we define the function spaces:

$$
\begin{aligned}
& X=\left\{\bar{u} \in\left(H_{1}(\Omega)\right) \mid \bar{u}=a \text { on } \Gamma_{\text {in }}, \bar{u}=0 \text { on } \Gamma_{\text {wall }}\right\} \\
& Q=\left\{\bar{u} \in\left(H_{1}(\Omega)\right) \mid \bar{u}=0 \text { on } \Gamma_{\text {in }} \cup \Gamma_{\text {wall }}\right\}
\end{aligned}
$$

The weak form of Eqns. (28) - (31) is obtained by determining $\mathrm{w} \in \mathrm{X}$ and $\phi, \theta \in \mathrm{P}$ such that every $v \in Q$ and $q \in P$ where $P=L^{2}(\Omega)$. Therefore, the weak formulation of Eqns. (28) - (31) will be:

$$
\begin{gathered}
\int_{\Omega} \frac{c A}{S B^{2}} \frac{\partial \bar{\theta}}{\partial \bar{z}} \frac{\partial \bar{w}}{\partial \bar{r}} \cdot u d r-\int_{\Omega} \lambda_{1} \frac{1}{\bar{r}}\left(\frac{\partial \bar{w}}{\partial \bar{r}}\right)^{2} \cdot u d r-\int_{\Omega} 2 \frac{\partial \bar{w}}{\partial \bar{r}} \frac{\partial^{2} \bar{w}}{\partial \bar{r}^{2}} \cdot u d r \\
-\int_{\Omega} B_{r} \bar{\phi} \cdot u d r-\int_{\Omega} G_{r} \bar{\theta} \cdot u d r=0 \\
\int_{\Omega} \frac{c A}{S B^{2}} \frac{\partial \bar{\theta}}{\partial \bar{r}} \frac{\partial \bar{w}}{\partial \bar{r}} \cdot v d r-\int_{\Omega} \lambda_{1} \frac{\partial \bar{w}}{\partial \bar{z}} \frac{\partial^{2} \bar{w}}{\partial r^{2}} \cdot v d r-\int_{\Omega} \lambda_{1} \bar{w} \frac{\partial^{3} \bar{w}}{\partial \bar{r}^{2} \partial \bar{z}} \cdot v d r \\
-\int_{\Omega} \lambda_{1} \frac{\bar{w}}{\bar{r}} \frac{\partial^{2} \bar{w}}{\partial \bar{r} \partial \bar{z}} \cdot v d r-\int_{\Omega} \lambda_{1} \frac{1}{\bar{r}} \frac{\partial \bar{w}}{\partial \bar{r}} \frac{\partial \bar{w}}{\partial \bar{z}} \cdot v d r-\int_{\Omega} \lambda_{1} \frac{\partial \bar{w}}{\partial \bar{r}} \frac{\partial^{2} \bar{w}}{\partial \bar{r} \partial \bar{z}} \cdot v d r \\
-\int_{\Omega} 2 \lambda_{1} \frac{\partial \bar{w}}{\partial \bar{r}} \frac{\partial^{2} \bar{w}}{\partial \bar{z} \partial \bar{r}} \cdot v d r-\int_{\Omega} G_{r} \bar{\theta} \cdot v d r-\int_{\Omega} B_{r} \bar{\phi} \cdot v d r+\int_{\Omega} M^{2} \bar{w} \cdot v d r=0 \\
\int_{\Omega} \frac{\partial^{2} \bar{\theta}}{\partial \bar{r}^{2}} \cdot w d r+\int_{\Omega} \frac{1}{\bar{r}} \frac{\partial \bar{\theta}}{\partial \bar{r}} \cdot w d r+\int_{\Omega} N_{b}\left[\frac{\partial \bar{\phi}}{\partial \bar{r}} \frac{\partial \bar{\theta}}{\partial \bar{r}}\right] \cdot w d r+\int_{\Omega} N_{t}\left[\frac{\partial \bar{\theta}}{\partial \bar{r}} \frac{\partial \bar{\theta}}{\partial \bar{r}}\right] \cdot w d r=0 \\
\int_{\Omega} \frac{\partial^{2} \bar{\phi}}{\partial \bar{r}^{2}} \cdot q d r+\int_{\Omega} \frac{1}{\bar{r}} \frac{\partial \bar{\phi}}{\partial \bar{r}} \cdot q d r+\int_{\Omega} \frac{N_{t}}{N_{b}}\left(\frac{\partial^{2} \bar{\theta}}{\partial \bar{r}^{2}}+\frac{1}{\bar{r}} \frac{\partial \bar{\theta}}{\partial \bar{r}}\right) \cdot q d r=0
\end{gathered}
$$

A fundamental aspect of the current modelling is to obtain the weak form of the above system of Eqns. (36)-(39). To achieve smoothness of the solution which is bounded due to the weaker restriction, these differential equations cannot be solved directly. So the finite dimensional subspaces have to be defined as $Q_{h} \subset Q$ and $P_{h} \subset P$. Consider the finite dimensional approximations as $u_{h}, v_{h} \subset Q_{h}$ and $w_{h}, q_{h} \subset P_{h}$. In view of the finite dimensional approximation, the set of Eqns. (36)-(39) becomes: 


$$
\begin{gathered}
\int_{\Omega} \frac{c A}{S B^{2}} \frac{\partial \bar{\theta}}{\partial \bar{z}} \frac{\partial \bar{w}}{\partial \bar{r}} \cdot u_{h} d r-\int_{\Omega} \lambda_{1} \frac{1}{\bar{r}}\left(\frac{\partial \bar{w}}{\partial \bar{r}}\right)^{2} \cdot u_{h} d r-\int_{\Omega} 2 \frac{\partial \bar{w}}{\partial \bar{r}} \frac{\partial^{2} \bar{w}}{\partial \bar{r}^{2}} \cdot u_{h} d r \\
\quad-\int_{\Omega} B_{r} \bar{\phi} \cdot u_{h} d r-\int_{\Omega} G_{r} \bar{\theta} \cdot u_{h} d r=0 \\
\int_{\Omega} \frac{c A}{S B^{2}} \frac{\partial \bar{\theta}}{\partial \bar{r}} \frac{\partial \bar{w}}{\partial \bar{r}} \cdot v_{h} d r-\int_{\Omega} \lambda_{1} \frac{\partial \bar{w}}{\partial \bar{z}} \frac{\partial^{2} \bar{w}}{\partial r^{2}} \cdot v_{h} d r-\int_{\Omega} \lambda_{1} \bar{w} \frac{\partial^{3} \bar{w}}{\partial \bar{r}^{2} \partial \bar{z}} \cdot v_{h} d r \\
-\int_{\Omega} \lambda_{1} \frac{\bar{w}}{\bar{r}} \frac{\partial^{2} \bar{w}}{\partial \bar{r} \partial \bar{z}} \cdot v_{h} d r-\int_{\Omega} \lambda_{1} \frac{1}{\bar{r}} \frac{\partial \bar{w}}{\partial \bar{r}} \frac{\partial \bar{w}}{\partial \bar{z}} \cdot v_{h} d r-\int_{\Omega} \lambda_{1} \frac{\partial \bar{w}}{\partial \bar{r}} \frac{\partial^{2} \bar{w}}{\partial \bar{r} \partial \bar{z}} \cdot v_{h} d r \\
-\int_{\Omega} 2 \lambda_{1} \frac{\partial \bar{w}}{\partial \bar{r}} \frac{\partial^{2} \bar{w}}{\partial \bar{z} \partial \bar{r}} \cdot v_{h} d r-\int_{\Omega} G_{r} \bar{\theta} \cdot v_{h} d r-\int_{\Omega} B_{r} \bar{\phi} \cdot v_{h} d r+\int_{\Omega} M^{2} \bar{w} \cdot v_{h} d r=0 \\
\int_{\Omega} \frac{\partial^{2} \bar{\theta}}{\partial \bar{r}^{2}} \cdot w_{h} d r+\int_{\Omega} \frac{1}{\bar{r}} \frac{\partial \bar{\theta}}{\partial \bar{r}} \cdot w_{h} d r+\int_{\Omega} N_{b}\left[\frac{\partial \bar{\phi}}{\partial \bar{r}} \frac{\partial \bar{\theta}}{\partial \bar{r}}\right] \cdot w_{h} d r \\
+\int_{\Omega} N_{t}\left[\frac{\partial \bar{\theta}}{\partial \bar{r}} \frac{\partial \bar{\theta}}{\partial \bar{r}}\right] \cdot w_{h} d r=0 \\
\int_{\Omega} \frac{\partial^{2} \bar{\phi}}{\partial \bar{r}^{2}} \cdot q_{h} d r+\int_{\Omega} \frac{1}{\bar{r}} \frac{\partial \bar{\phi}}{\partial \bar{r}} \cdot q_{h} d r+\int_{\Omega} \frac{N_{t}}{N_{b}}\left(\frac{\partial^{2} \bar{\theta}}{\partial \bar{r}^{2}}+\frac{1}{\bar{r}} \frac{\partial \bar{\theta}}{\partial \bar{r}}\right) \cdot q_{h} d r=0
\end{gathered}
$$

Eqns. (40)-(43) with boundary conditions (32) are solved numerically using the variational finite element method in FreeFEM++ [68, 69]. In the present study we consider classic Taylor-Hood triangular elements $\left(P_{1}, P_{2}\right)$. The simulation features 5928 unstructured fixed mesh triangular elements with 12177 nodes as presented in Figure 2. The mesh is built taking advantage of an automatic FreeFEM++ mesh generator based on the Delaunay-Voronoi algorithm. The non-linear system of the governing equations has been solved by employing the Generalized Minimal Residual (GMRES) iteration method. In the fixed mesh the prescribed minimum step size $\left(h_{\min }\right)$ is 0.0001 and the tolerance for computation is $<10^{-6}$ for all simulations.

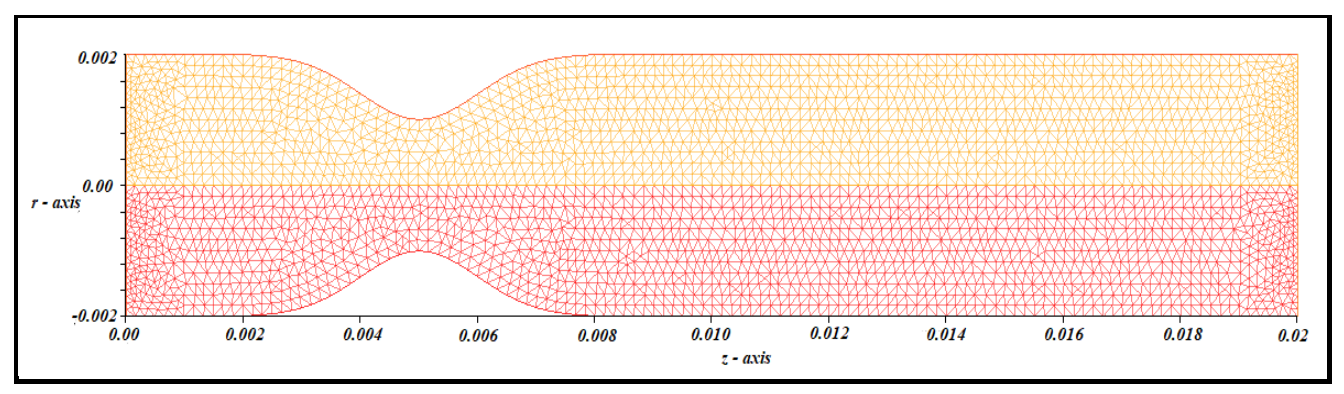

Figure 2. Unstructured fixed mesh of triangular elements 


\section{GRID SENSITIVITY ANALYSIS}

To ensure that the calculated numerical results are grid-independent, several different grid distributions have been tested. Table 1 shows the numerical values for velocity, temperature and nano-particle concentration for various designs of unstructured fixed mesh elements involving vertices and triangular elements as shown in Figure 2. Several different mesh distributions have been tested to ensure that the simulated numerical results are mesh independent. Therefore, the selected mesh for the present calculations consisted of 12177 nodes and 5928 triangular elements respectively. It can be seen from Table 1 along with figures 3 and 4 that increasing the mesh elements beyond this design does not modify significantly the numerical values of nondimensional velocity, temperature, nano-particle concentration and skin-friction coefficient in the domain with the parametric values prescribed as $M=0.3, N_{b}=0.3, N_{t}=0.3, \lambda_{1}=0.5, A=B=5$, $d=0.4 l, r=0.0001, z=0.005$ and $c=0.3$. Mesh-independent results are therefore ensured with the mesh design comprising 12177 nodes and 5928 triangular elements (simulation number 4 in Table 1).

Table 1. Grid Independency analysis with $M=0.3, N_{b}=0.3, N_{t}=0.3, \lambda_{1}=0.5, A=B=5, \operatorname{Re}=1$ $d=0.4 l, r=0.0001, z=0.005$ and $c=0.3$

\begin{tabular}{|c|c|c|c|c|c|c|}
\hline $\begin{array}{c}\text { Simulation } \\
\text { No. }\end{array}$ & $\begin{array}{c}\text { No. of } \\
\text { Nodes }\end{array}$ & $\begin{array}{c}\text { No. of } \\
\text { elements } \\
(\text { Cells })\end{array}$ & $\begin{array}{c}\text { Velocity } \\
(\mathbf{w})\end{array}$ & $\begin{array}{c}\text { Temperature } \\
(\boldsymbol{\theta})\end{array}$ & $\begin{array}{c}\text { Nanoparticles } \\
\text { Concentration } \\
(\boldsymbol{\phi})\end{array}$ & $\begin{array}{c}\text { Skin-friction } \\
\text { coefficient } \\
(\mathbf{a t} \mathbf{r}=\mathbf{0 . 0 0 1}) \\
\left(\frac{1}{2} C_{f} \operatorname{Re}\right)\end{array}$ \\
\hline 1. & 7949 & 3854 & 0.98486 & 0.0526 & 0.0526 & 0.89326 \\
\hline 2. & 8389 & 4064 & 0.98765 & 0.053498 & 0.053497 & 0.97221 \\
\hline 3. & 11201 & 5460 & 0.98842 & 0.063114 & 0.063114 & 0.99551 \\
\hline $\mathbf{4 .}$ & $\mathbf{1 2 1 7 7}$ & $\mathbf{5 9 2 8}$ & $\mathbf{0 . 9 9 6 1 7}$ & $\mathbf{0 . 0 6 7 0 7 3}$ & $\mathbf{0 . 0 6 7 0 7 3}$ & $\mathbf{1 . 0 1 4 2}$ \\
\hline 5. & 24909 & 12244 & 0.99671 & 0.068061 & 0.068062 & 1.01693 \\
\hline 6. & 27261 & 13390 & 0.99678 & 0.069895 & 0.06989 & 1.0136 \\
\hline 7. & 50837 & 25098 & 0.99715 & 0.071355 & 0.071352 & 1.036 \\
\hline
\end{tabular}




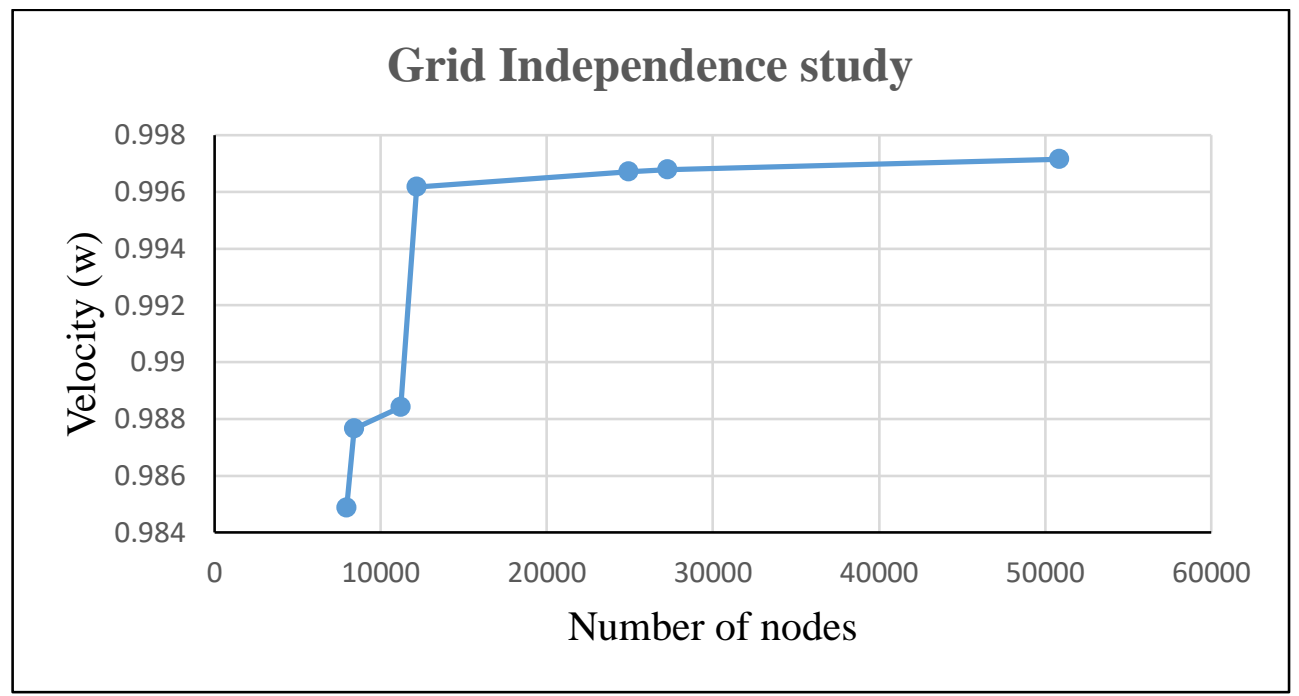

Figure 3: Grid independence study (Velocity)

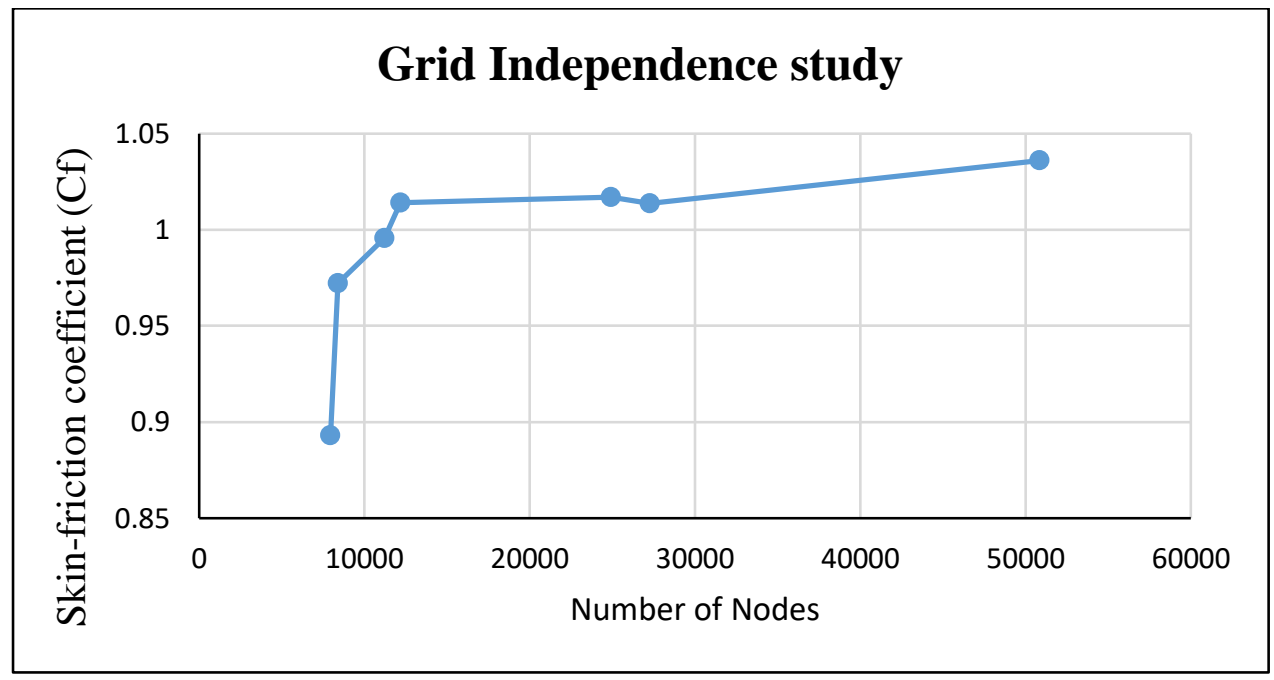

Figure 4: Grid independence study (Skin-friction coeff.)

\section{RESULTS AND DISCUSSION}

In this section the response in velocity, temperature and nano-particle concentration in the magnetic stenotic hemodynamic flow is studied for different shape and designs of stenosis and also with variation of different parameters. For computation of the model, the default values of different parameters are given in Table 2.

Table 2: Default values of key parameters

\begin{tabular}{|l|l|l|l|l|l|l|l|l|l|l|l|l|l|l|l|}
\hline Parameter & $\mu_{0}$ & $\theta_{0}$ & $G_{r}$ & $B_{r}$ & $L$ & $l$ & $M$ & $N_{b}$ & $N_{t}$ & $\lambda_{1}$ & $d$ & $A$ & $B$ & $c$ & $R e$ \\
\hline Values & 0.5 & 0.5 & 1.0 & 1.0 & 0.020 & 0.005 & 0.3 & 0.3 & 0.3 & 0.5 & $0.4 l$ & 5 & 5 & 0.3 & 3 \\
\hline
\end{tabular}


The visualizations of non-dimensional velocity profile for different values of magnetic parameter $(M)$ and thermophoresis parameter $\left(N_{t}\right)$ is documented in figures 5(a), 5(b), 5(c) and 5(d) in the arterial domain, $\Omega$, corresponding to the mild stenosis case (here the blood flow direction is taken from left to right).

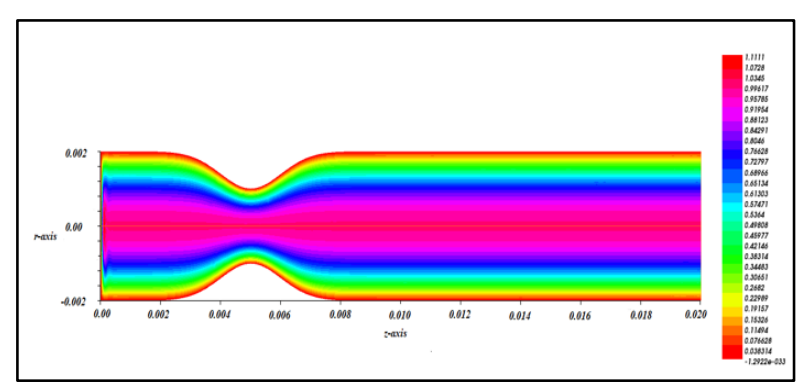

5(a)

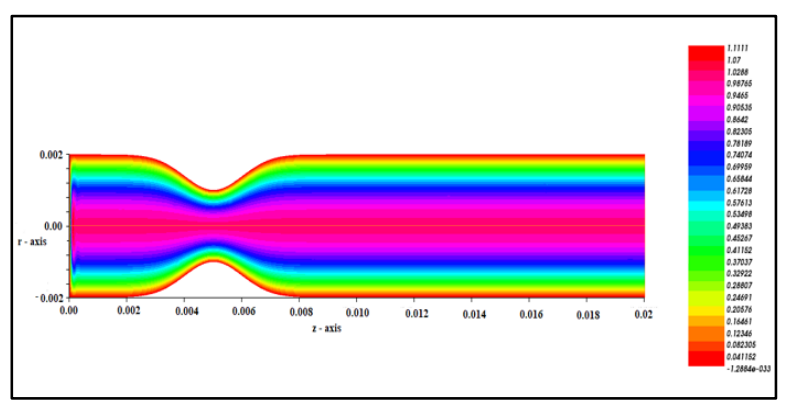

5(c)

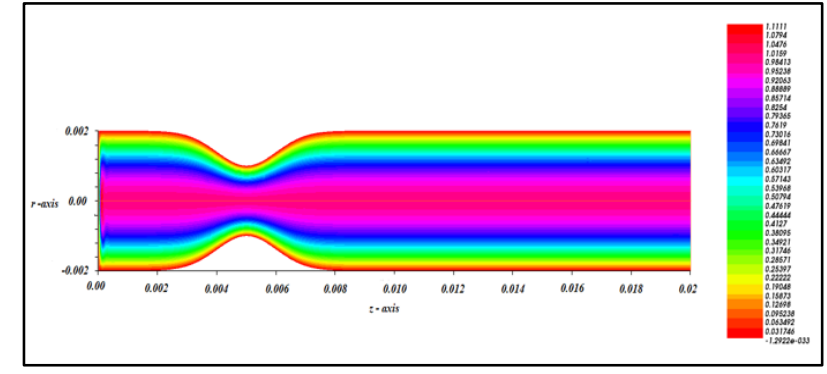

5(b)

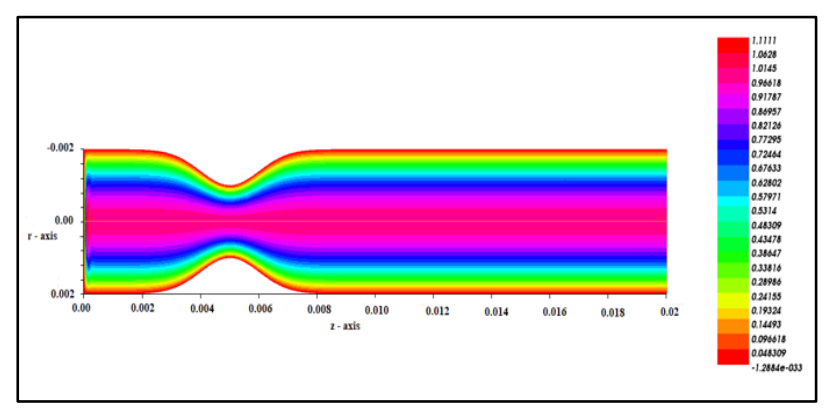

5(d)

Figure 5 Velocity field (a) $M=0.3, N_{b}=0.3$ and $N_{t}=0.3$ (b) $M=0.3, N_{b}=0.3$ and $N_{t}=0.6$, (c) $M=0.6, N_{b}=0.3$ and $N_{t}=0.3$, (d) $M=0.6, N_{b}=0.3$ and $N_{t}=0.6$.

It is observed from figures 5(a)-5(b), that increasing the values of thermophoresis parameter $\left(N_{t}\right)$ from 0.3 to 0.6 (with magnetic parameter, $M$, and Brownian motion parameter, $N_{b}$ fixed) decreases the value of velocity in whole domain. Thermophoresis relates to the migration of nano-particles under the force of a temperature gradient. With nano-particles migrating the momentum is inhibited in the arterial flow and deceleration induced. Effectively thermophoretic body force arises due to the averaged Brownian motion of particles in the nano-doped blood under a steady temperature gradient. Since steady state conditions are assumed, the stronger molecular impulses in the hotter nanofluid region mobilize nano-particles to migrate towards the colder region, where the molecular impulses are weaker. This destroys momentum in the blood flow and results in a depletion in velocity. In particular, in the core zone strong deceleration is induced. With increasing magnetic parameter $(M)$ from 0.3 to 0.6 as shown in figures $5 \mathrm{a}$ and $5 \mathrm{c}$ (for $N_{t}=0.3$ ) and figures $5 \mathrm{~b}$ and $5 \mathrm{~d}$ (for $N_{t}=0.6$ ) there is also a significant decrement in velocity i.e. the core flow is again retarded. The presence of the Lorentzian magnetic drag force resists blood flow and especially generates deceleration in the constricted zone at the stenosis which in turn influences the remainder of the flow domain. In all plots, 
the velocity is maximum at centreline of the artery and decreases to the boundary at which it vanishes in accordance with the no-slip condition.

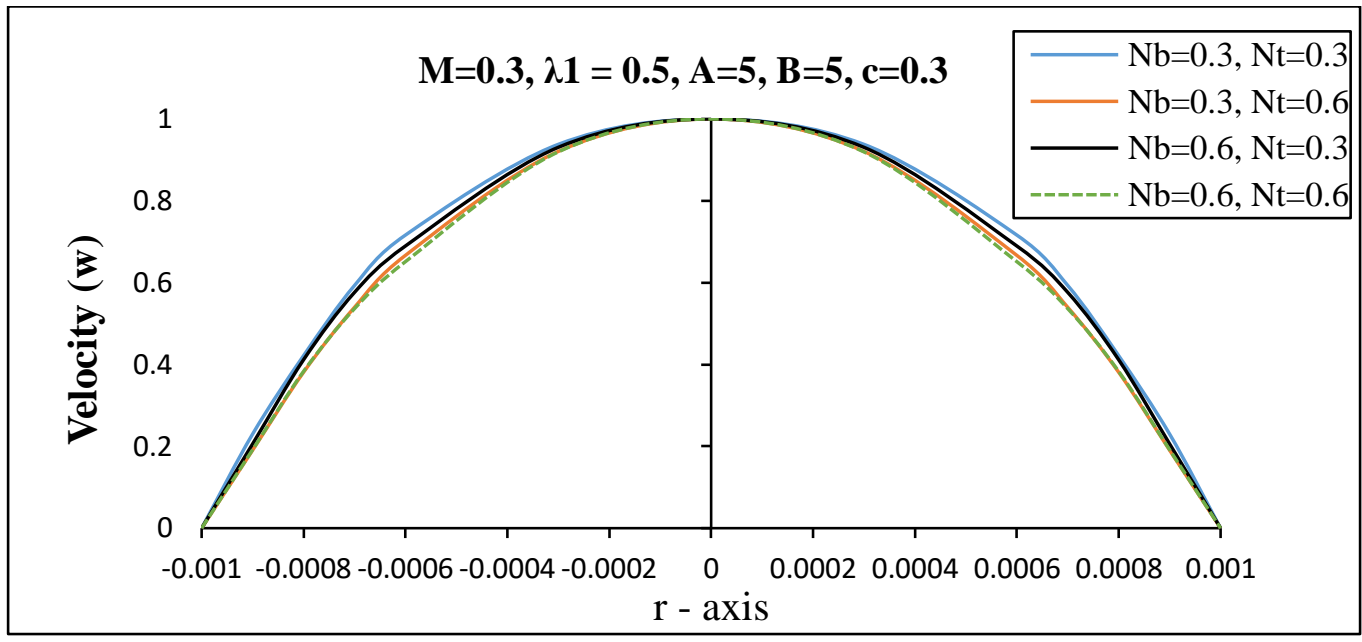

Figure 6 Velocity profile for diameter of stenosis $=0.4 l$ at $z=0.005$

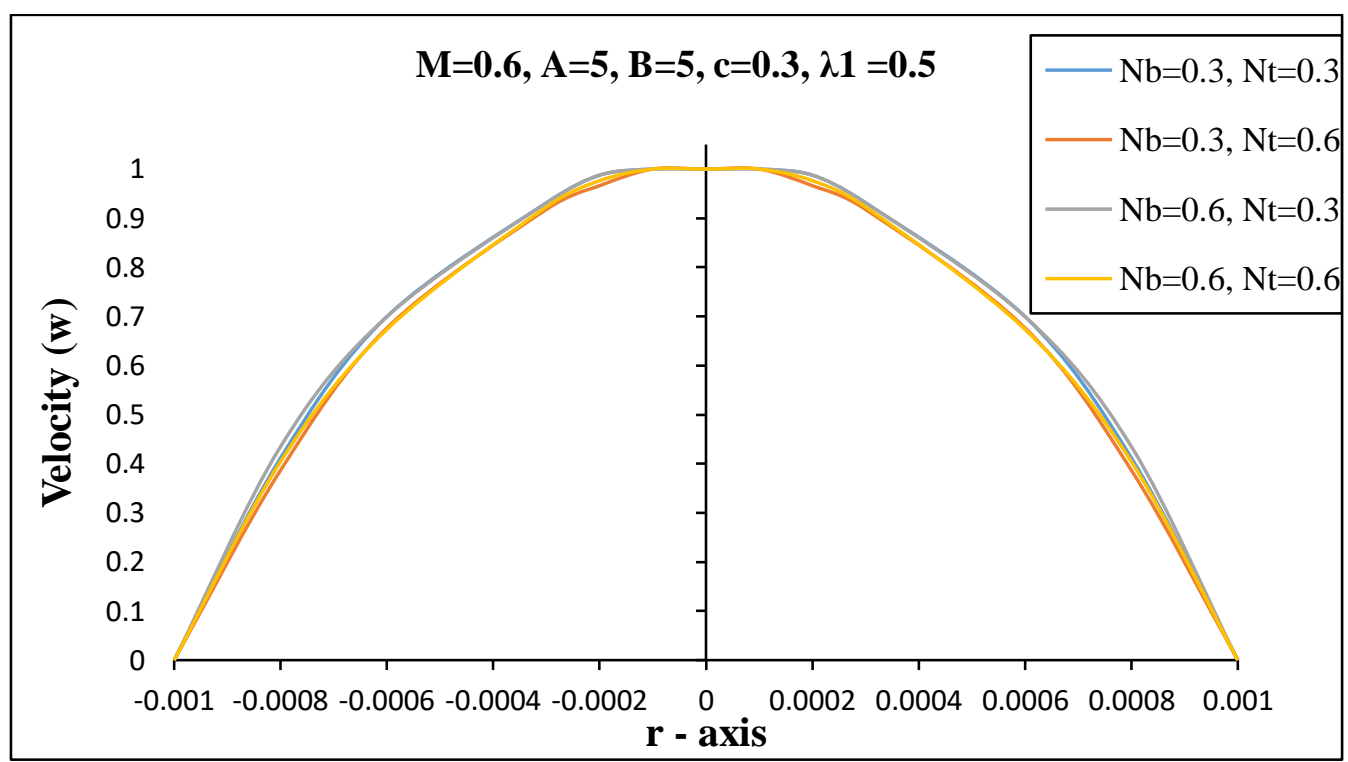

Figure 7 Velocity profile for diameter of stenosis $=0.4 l$ at $z=0.005$ 


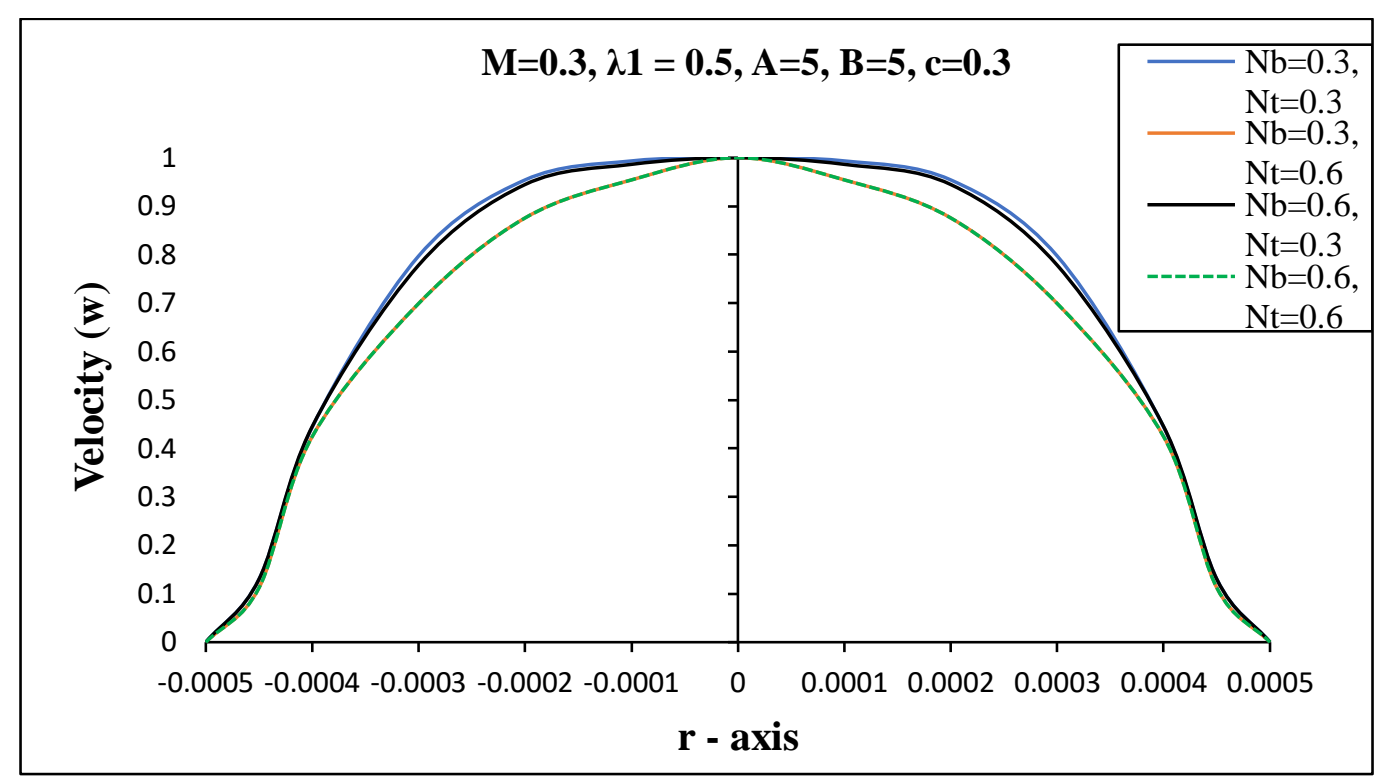

Figure 8 Velocity profile for diameter of stenosis $=0.3 \mathrm{l}$ at $z=0.005$

Figures 6, 7 and 8 illustrate the velocity profiles at a specific location of the arterial segment $\mathrm{z}=0.005$ in the stenotic region (the throat of the stenosis) with variation along the $r-$ axis for selected values of thermophoresis parameter $\left(N_{t}\right)$ and Brownian motion parameter $\left(N_{b}\right)$. In these plots the magnetic parameter is also varied although it is constrained to be less than unity indicating that the Lorentzian drag force is always less in magnitude than the viscous hemodynamic force in the regime. Figure 8 corresponds to the velocity profile when the diameter of the stenosis, $d=0.3 l$. It can be seen from the above figures that increasing the magnetic parameter clearly results in a significant reduction in velocity magnitudes. The imposition of a transverse magnetic field, as elaborated earlier, has a tendency to develop impedance known as the Lorentz drag force which acts perpendicular to the magnetic field i.e. along the axial direction. Stronger magnetic field corresponds to higher $M$ values and this manifests in blood flow deceleration. However, by increasing the Brownian motion parameter $\left(N_{b}\right)$, the velocity is observed to increase. Larger values of $N_{b}$ are associated with physically smaller nanoparticles in the Buongiorno model. This encourages ballistic collisions and momentum diffusion in the hemodynamic flow which results in flow acceleration. The decelerating effect of increasing thermophoresis parameter, $N_{t}$, already observed in Figures 5(a)-(d) is confirmed in figures 6-8. There is a marked depression computed in velocity magnitudes and this is observed across the arterial span i.e. for all radial coordinate values. 


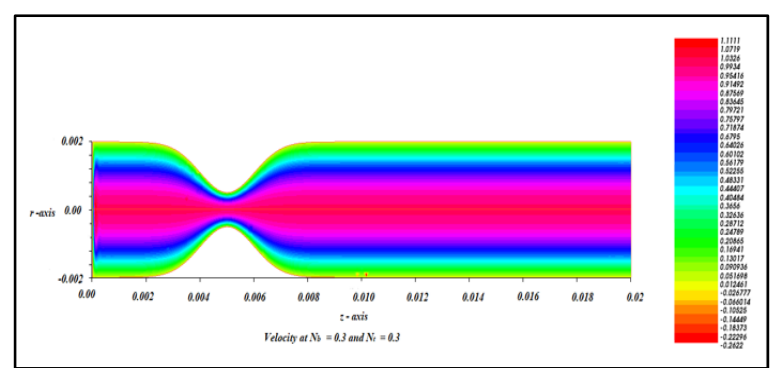

9(a)

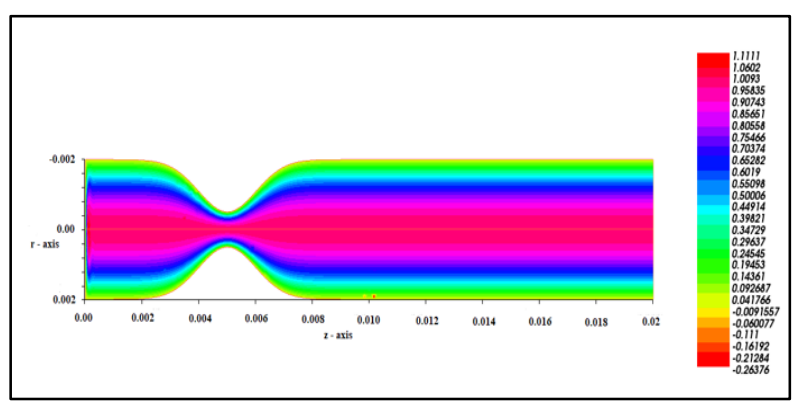

9(c)

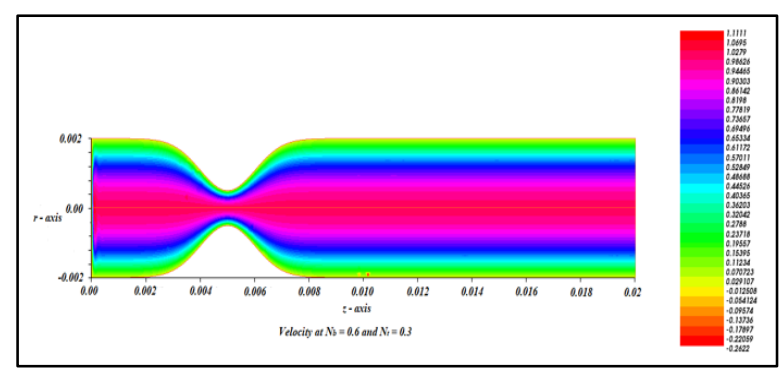

9(b)

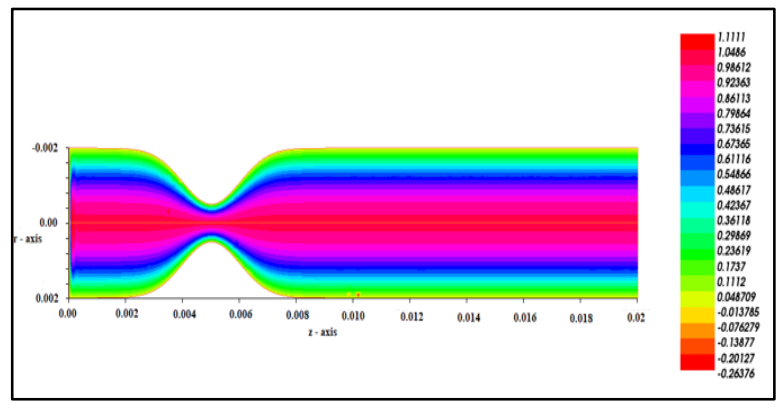

9(d)

Figure 9 Velocity contour plots (a) $M=0.3, N_{b}=0.3$ and $N_{t}=0.3$ (b) $M=0.3, N_{b}=0.6$ and $N_{t}=0.3$, (c) $M=0.6, N_{b}=0.3$ and $N_{t}=0.3$ (d) $M=0.6, N_{b}=0.6$ and $N_{t}=0.3$.

Figs. 9(a)-(d) represents the non-dimensional velocity profile for another size of stenosis with different values of magnetic parameter $(M)$ and Brownian motion parameter $\left(N_{b}\right)$. The figure indicates that the radial velocity is progressively decreasing with greater values of $M$, associated with the related enhancement in Lorentz drag force. Significant impedance to blood flow is therefore clearly induced with stronger applied magnetic field and this is immensely useful in biomagnetic therapies for arterial diseases. It is also apparent that velocity is elevated by increasing the Brownian motion parameter due to the associated exacerbation in nano-particle collisions when they are smaller in size. Higher velocity contours (dark red) are observed to replace the lower velocity (pink) contours in the constricted zone from fig. 9c to $9 \mathrm{~d}$ for which the $N_{b}$ value is doubled from 0.3 to 0.6 with $M$ constrained at 0.6 and $N_{t}$ fixed at 0.3 .

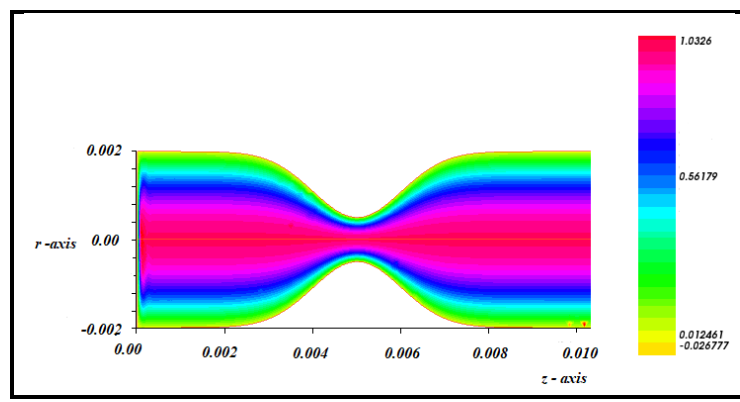

10(a)

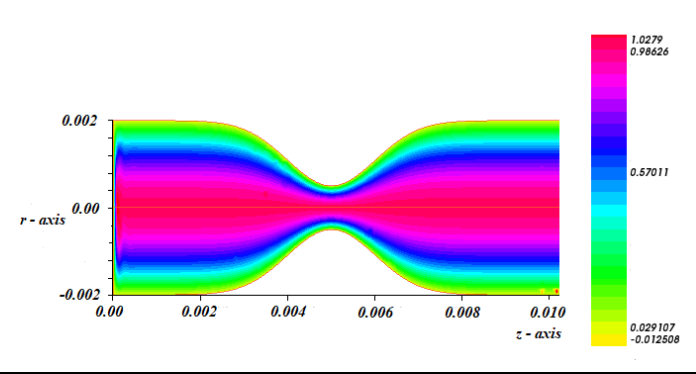

10(b)

Figure 10 Velocity contours for (a) $M=0.3, N_{b}=0.3$ and $N_{t}=0.3$ (b) $M=0.3, N_{b}=0.6$ and $N_{t}=0.3$ 
Figure 10(a) and 10(b) showing the affected part of the above two figures 9(a) and 9(b) with stenosis diameter $(\mathrm{d}=0.3 l)$. The increment in velocity by increasing the Brownian motion parameter $\left(N_{b}\right)$ is verified from inspection of the contours clearly. Smaller nanoparticles (higher $N_{b}$ ) values therefore successfully induce acceleration in the blood flow.

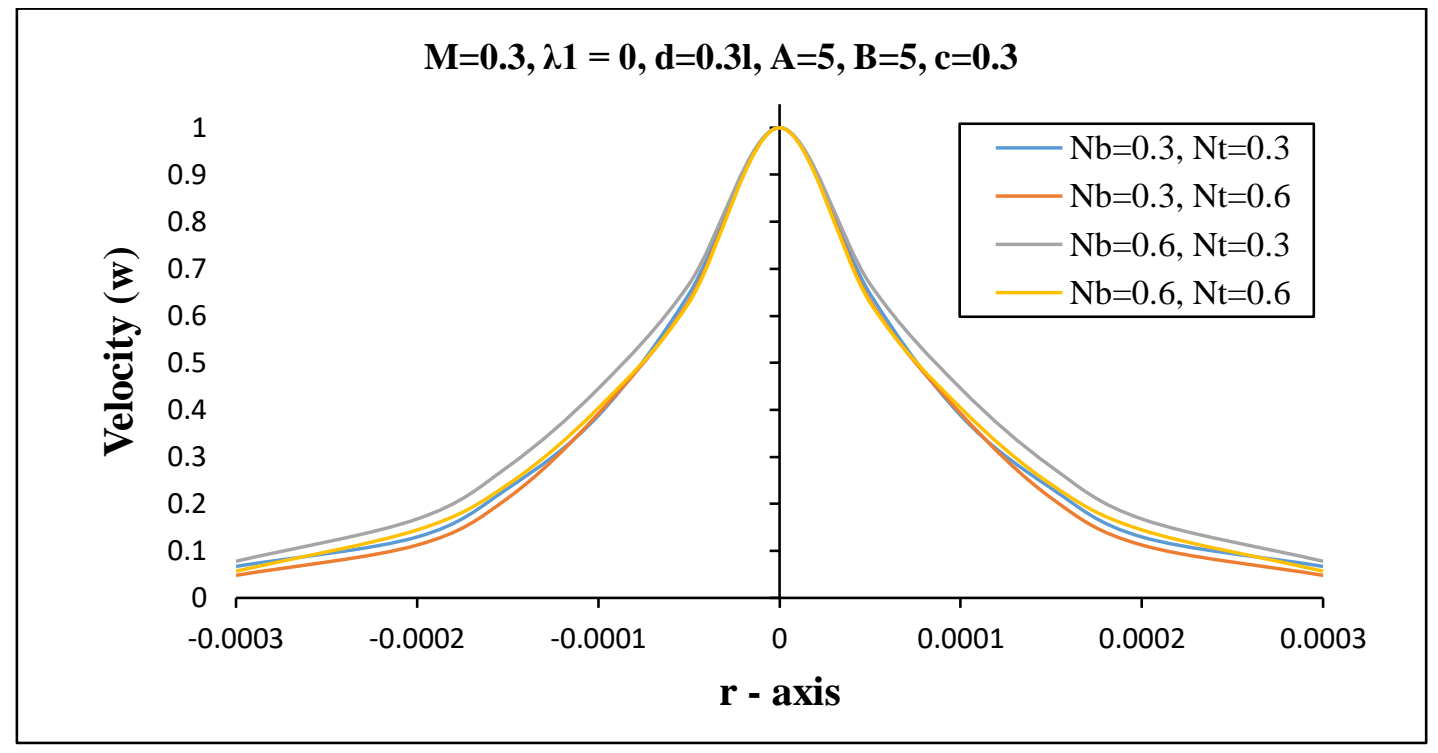

Figure 11 Velocity profile for diameter of stenosis $=0.3 l$ at $z=0.005$

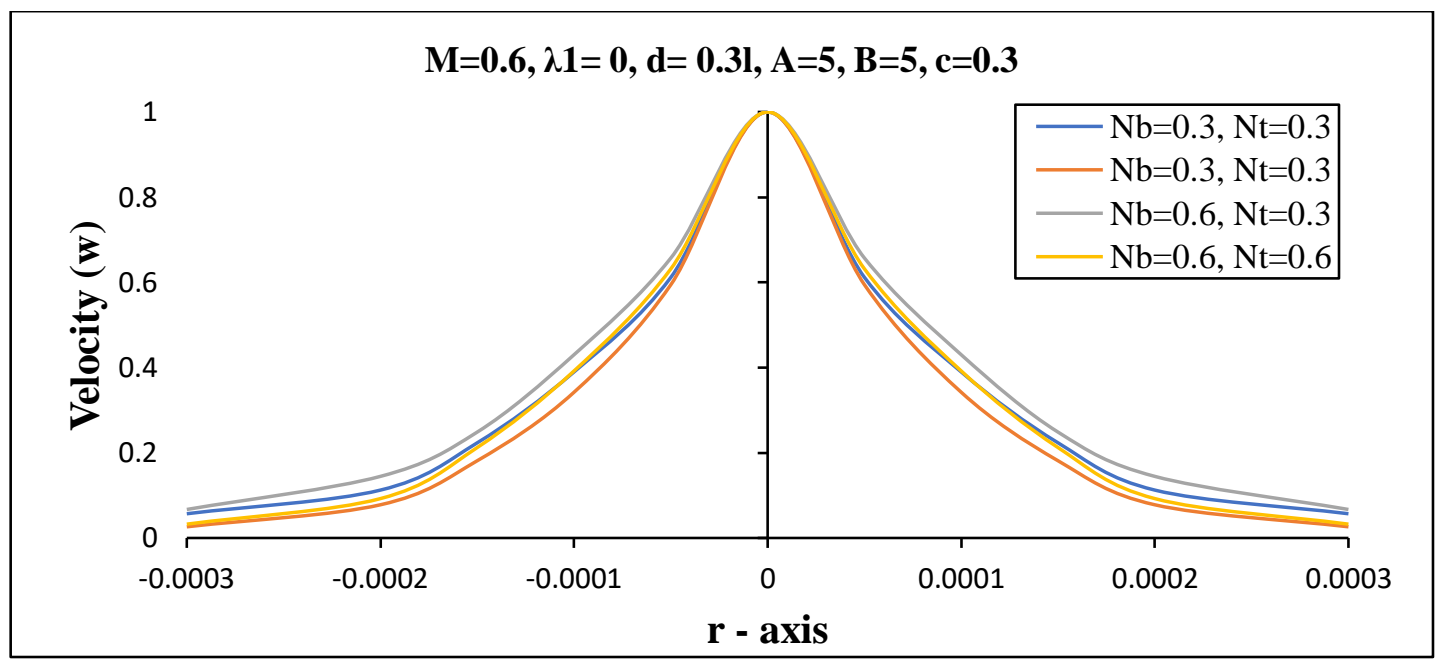

Figure 12 Velocity profile for diameter of stenosis $=0.3 l$ at $z=0.005$

Figs. 11 and 12 depict the evolution in dimensionless velocity profiles for different values of $N_{t}$ and $N_{b}$ when the blood is treated as Newtonian fluid i.e., $\lambda_{1}=0$ for the second stenosis case (stenosis diameter $d=0.3 l$ ) with variation of thermophoresis parameter $\left(N_{t}\right)$, Brownian motion parameter $\left(N_{b}\right)$, and magnetic body force parameter. Again, it is evident that blood velocity increases with increasing Brownian motion parameter $\left(N_{b}\right)$, whereas it is markedly depleted with 
increasing thermophoresis parameter $\left(N_{t}\right)$. The nanoscale effects are therefore dual in nature. Brownian dynamics is found to accelerate the stenotic flow whereas thermophoretic body force induces retardation in the stenotic flow.

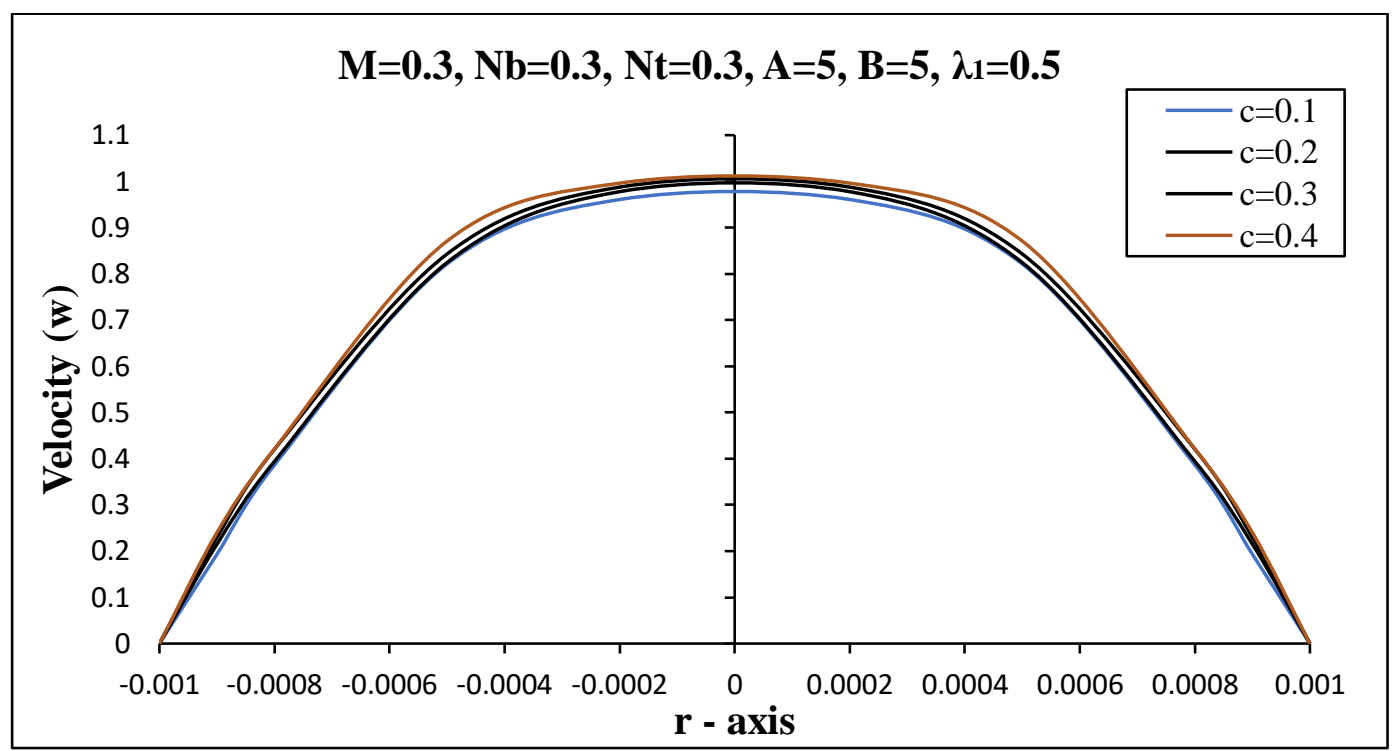

Figure 13 Velocity profile for diameter of stenosis $=0.4 l$ at $z=0.005$

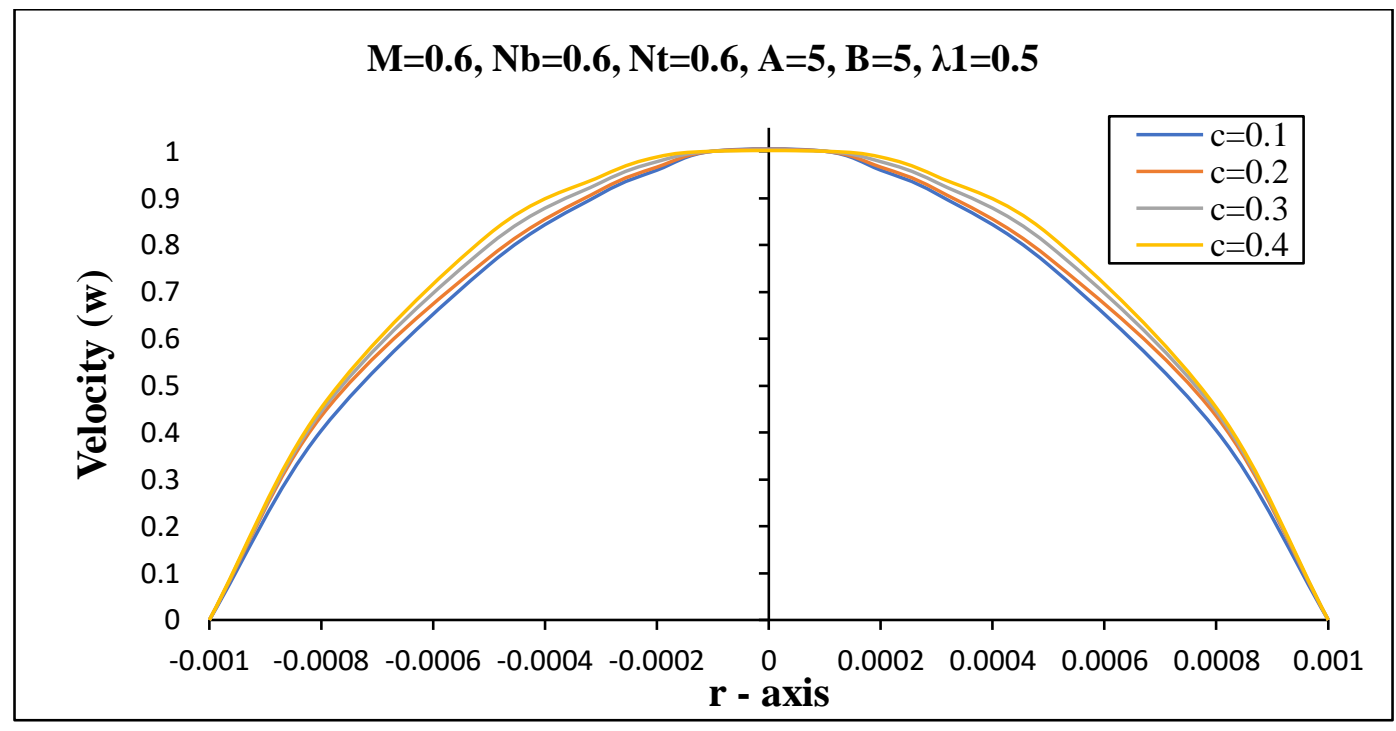

Figure 14 Velocity profile for diameter of stenosis $=0.4 l$ at $z=0.005$

Figs. 13 and 14 illustrate the variation in blood velocity profile with the variation of Vogel viscosity coefficient, $c$, for two values of magnetic body force parameter, $M$. Absolute viscosity of the blood as defined by Vogel's model in Eqn. (27), $\bar{\mu}=\frac{c}{S}\left[1-\frac{A \bar{\theta}}{B^{2}}\right]$, is directly proportional to the parameter, $c$. As this parameter is increased the viscosity is elevated which modifies the velocity field. Blood flow is observed to be weakly accelerated with increasing Vogel parameter, $c$. 
Additionally the velocities computed in Fig. 13 are observed to be larger than those in Fig. 14 since the magnetic parameter, $M$, is doubled from 0.3 to 0.5 . This doubles the Lorentz magnetic drag force and morphs the profiles from a more plateau-like distribution across the vessel to a sharper parabolic profile with lower magnitudes. The velocity around the core region i.e. juxtapose to the mid-line $(r=0)$ of the arterial vessel, is dramatically reduced, with greater magnetic body force parameter.

Fig. 15 shows the response in velocity field with variation in the Vogel's viscosity parameter $(A)$. This parameter arises both individually as a numerator and as an exponent in the denominator parameter, $S$, as defined in Eqn. (27). The overall effect is that viscosity is significantly modified and leads to a deceleration in the blood flow in the arterial vessel. This effect is clearly captured across the cross section of the vessel i.e. for all values of radial coordinate, $r$.

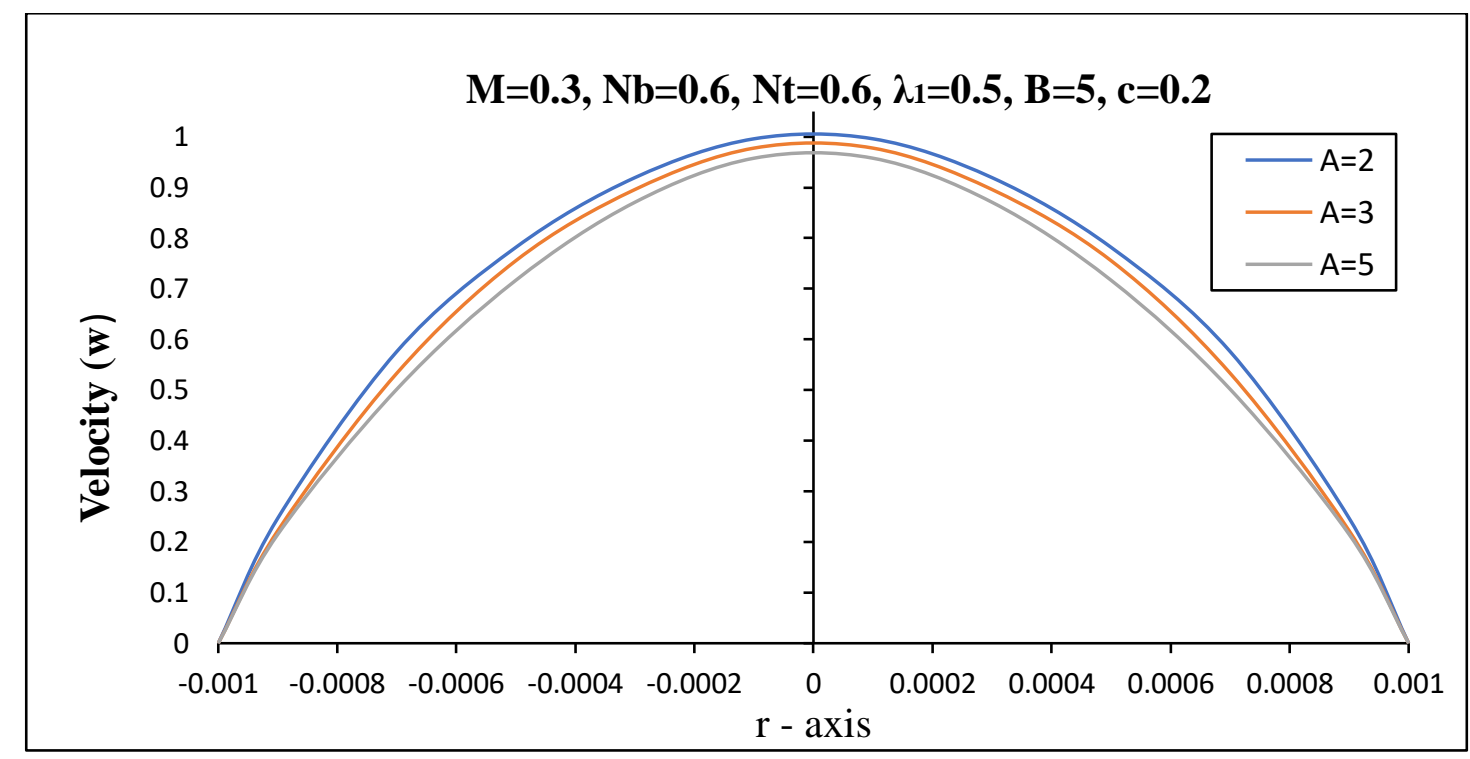

Figure 15 Velocity profile for diameter of stenosis of $0.4 l$ at $z=0.005$ 


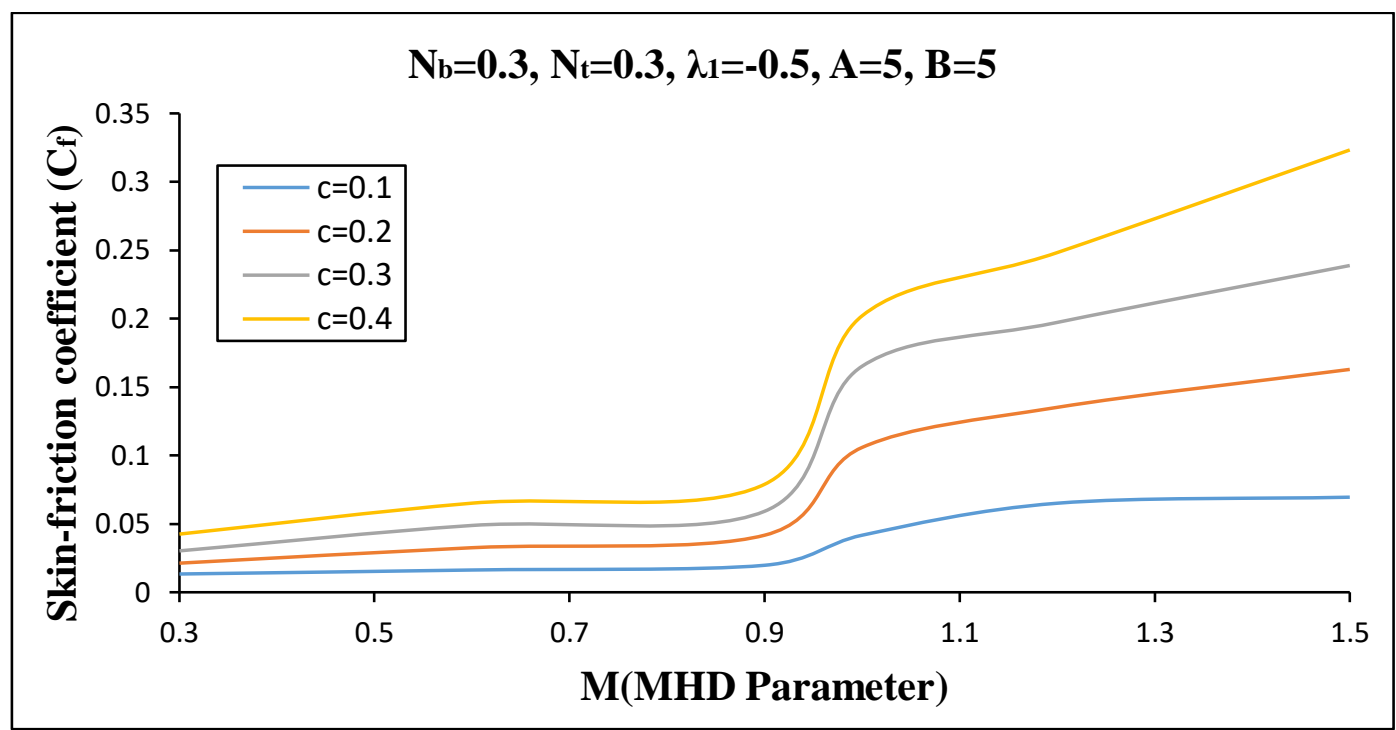

Figure 16 Skin-friction coefficient for diameter of stenosis of $0.4 l$ at $\boldsymbol{r}=0$

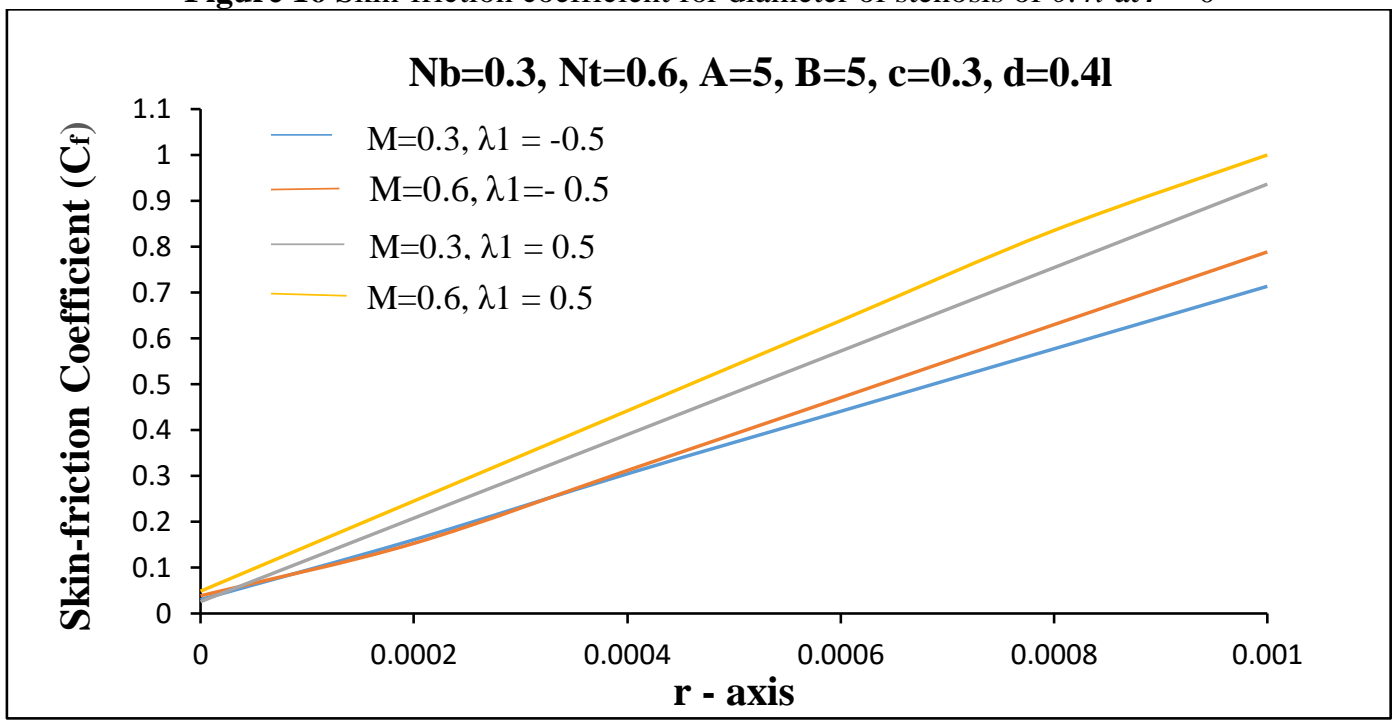

Figure 17 Skin-friction coefficient for diameter of stenosis $=0.4 l$ at $z=0$

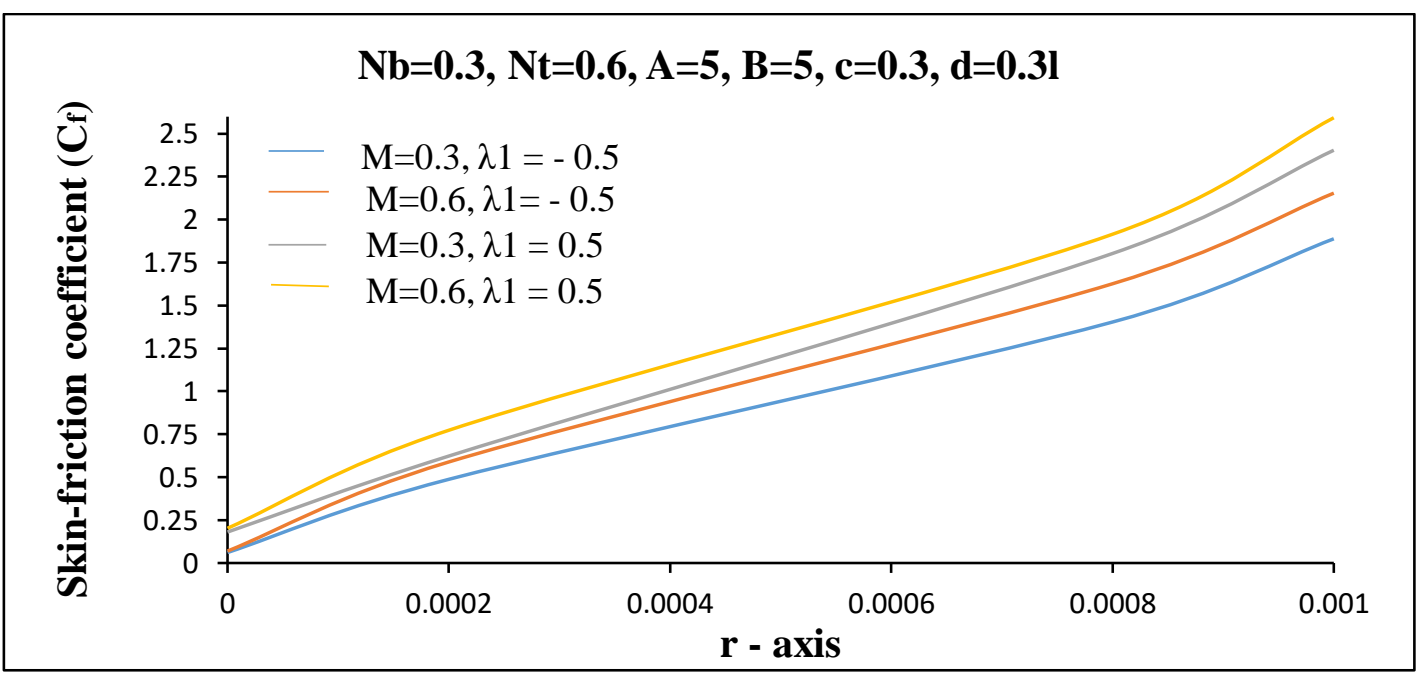

Figure 18 Skin-friction coefficient for diameter of stenosis $=0.3 l$ at $z=0$ 
Figure 16 illustrates the skin-friction coefficient distribution versus magnetic parameter $(M)$ for various values of the Vogel parameter, $c$, at the arterial mid-line $(r=0)$. It is evident that with greater $M$ values the skin friction is initially weakly increased up to $M=0.9$. However, subsequently, for $0.9<M<1.1$ there is a significantly sharper increase in skin friction and thereafter the ascent is less dramatic. Evidently when magnetic body force becomes equal to viscous hemodynamic force $(M=1)$ there is a step-up in the impact of magnetic field. Following this even though magnetic body force is increasingly greater than viscous force, the skin friction while continuing to increase does so less sharply. Consistently there is an elevation in blood flow velocity with Vogel parameter, $c$, for all values of magnetic parameter. However, the most dramatic enhancement clearly corresponds to the case when $M>0.9$.

Figs. 17 and 18 depicts the variation of skin-friction coefficient with radial coordinate $(r)$ for different magnetic parameter $(M)$ and viscoelastic parameter $\left(\lambda_{1}\right)$ values for two different cases of stenosis (i.e., stenosis diameter $d=0.4 l$ and $d=0.3 l$, respectively). It is apparent that when the artery is narrower (i.e., diameter of stenosis $d=0.3 l$ ) the value of skin friction coefficient is considerably greater (Fig. 17) than when the artery is wider (diameter of stenosis $d=0.4 l$.). In Fig. 17 the skin friction coefficient magnitudes are evidently much lower. The greater constriction corresponding to the narrower artery clearly impedes the flow and leads to higher wall shear stress (WSS) i.e. greater skin friction, which characterizes arterial disease. With positive viscoelastic parameter $\left(\lambda_{1}\right.$ ) values much higher skin friction magnitudes are computed (Fig. 17) than for negative viscoelastic parameter $\left(\lambda_{1}\right)$ values. This parameter arises in both the normalized radial and axial momentum conservation equations i.e. (28) and (29) in the terms $\lambda_{1}\left[\frac{1}{r}\left(\frac{\partial \bar{w}}{\partial \bar{r}}\right)^{2}+2 \frac{\partial \bar{w}}{\partial \bar{r}} \frac{\partial^{2} \bar{w}}{\partial \bar{r}^{2}}\right]$ and $\lambda_{1}\left(\frac{\partial \bar{w}}{\partial \bar{z}} \frac{\partial^{2} \bar{w}}{\partial \bar{r}^{2}}-\right.$ $\left.\bar{w} \frac{\partial^{3} \bar{w}}{\partial \bar{r}^{2} \partial \bar{z}}+\frac{\bar{w}}{\bar{r}} \frac{\partial^{2} \bar{w}}{\partial \bar{r} \partial \bar{z}}+\frac{1}{r} \frac{\partial \bar{w}}{\partial \bar{z}} \frac{\partial \bar{w}}{\partial \bar{r}}+\frac{\partial \bar{w}}{\partial \bar{r}} \frac{\partial^{2} \bar{w}}{\partial \bar{r} \partial \bar{z}}+2 \frac{\partial \bar{w}}{\partial \bar{r}} \frac{\partial^{2} \bar{w}}{\partial \bar{z} \partial \bar{r}}\right)$. Clearly there is strong coupling with the velocity fields. The impact of viscoelastic parameter $\lambda_{1}=\frac{\alpha_{1} U_{0}}{\mu_{0} R_{0}}$, is therefore considerable. This parameter is inversely proportional to the reference dynamic viscosity, $\mu_{0}$. For negative values of $\lambda_{1}$ the large terms in Eqns. (28) and (29) are reversed in polarity and corresponds to blood flow with opposite relaxation and retardation characteristics to those for positive $\lambda_{l}$. With increasing magnetic parameter, $M$, there is a sustained elevation in skin friction in both Figs. 17 and 18 at all values of radial coordinate, $r$. it is also noteworthy that for the wider artery i.e. larger stenosis (Fig. 
18) a consistently linear growth in skin friction is witnessed. However, for the narrower artery (Fig. 18) a sharper ascent is observed at lower radial coordinate values followed by a gentler gradient at intermediate values and then finally sharper ascent at highest radial coordinates.

Table 3 documents the variation in wall skin-friction coefficient for the variation of MHD parameter $(M)$ with Vogel's parameter $(c)$ for the wider case of stenosis (i.e., $d=0.41$ ). It is evident that skin-friction coefficient increases monotonically with an increase in Vogel's model parameter, $\mathrm{c}$ at the throat of the stenosis (on the wall of the artery). Furthermore, there is a marked increment in skin friction coefficient with greater magnetic parameter, $M$, indicating once again that there is significant flow modification at the arterial wall.

Table 3. Comparison of values of skin friction coefficient with different values of Vogel's parameter and MHD parameter for the wider case of stenosis when $N_{b}=0.3, N_{t}=0.3, A=B=5, \lambda_{1}=0.5, r=0.001$ and $z=0.005$

\begin{tabular}{|c|c|c|c|c|}
\hline \multirow{2}{*}{$\begin{array}{c}\text { MHD } \\
\text { parameter } \\
(\mathbf{M})\end{array}$} & \multicolumn{4}{|c|}{$\boldsymbol{d = 0 . 4 l}$} \\
\cline { 2 - 5 } & $\mathbf{c = 0 . 1}$ & $\mathbf{c = 0 . 2}$ & $\mathbf{c = 0 . 3}$ & $\mathbf{c = 0 . 4}$ \\
\hline $\boldsymbol{0 . 1}$ & 1.206 & 1.2707 & 1.4373 & 1.9694 \\
\hline $\boldsymbol{0 . 2}$ & 1.2156 & 1.6866 & 1.9687 & 2.6709 \\
\hline $\boldsymbol{0 . 3}$ & 1.6843 & 2.2926 & 2.992 & 3.3107 \\
\hline $\boldsymbol{0 . 4}$ & 1.9671 & 2.6693 & 3.1123 & 3.7003 \\
\hline $\boldsymbol{0 . 5}$ & 2.2919 & 3.0017 & 3.5012 & 4.049 \\
\hline $\boldsymbol{0 . 6}$ & 2.4186 & 3.317 & 3.8963 & 4.4207 \\
\hline $\boldsymbol{0 . 7}$ & 2.6668 & 3.7816 & 4.2579 & 4.89061 \\
\hline
\end{tabular}

Figures 19 and 20 present the variation of temperature $(\theta)$ at the throat of stenosis $(\mathrm{z}=0.005)$ for both cases of stenosis (wider and narrower artery respectively), with different Brownian motion ( $N_{b}$ ) and thermophoresis $\left(N_{t}\right)$ parameters. An inverse parabolic distribution is computed (in both figures) with vanishing temperature at the arterial mid-line i.e. $r=0$ with steady growth in temperature towards the walls of the artery where it is maximized. In both figures the value of temperature decreases across the arterial domain when Brownian motion parameter $\left(N_{b}\right)$ increases. This applies both at the stenosis and the boundary of vessel before and after the stenosis). Brownian motion therefore while encouraging momentum diffusion (it accelerates the flow as indicated in earlier graphs) is found to inhibit thermal diffusion which results in a cooling of the arterial regime. However, with increment of thermophoresis parameter $\left(N_{t}\right)$ there is a clear 
enhancement in temperatures across the arterial cross-section. Thermophoresis therefore has the opposite influence to Brownian motion i.e. it enhanced thermal diffusion and opposes momentum diffusion (inducing deceleration as noted earlier). It can be also be observed that in the second stenotic case i.e. narrower artery (Fig 20) the magnitudes of the temperature in the core region are substantially higher than for the first stenotic case i.e. wider artery (Fig. 19). Geometric stenotic effects therefore both influence velocity distribution (as shown earlier) and via coupling with the energy field will also impact on thermal diffusion in the blood flow regime.

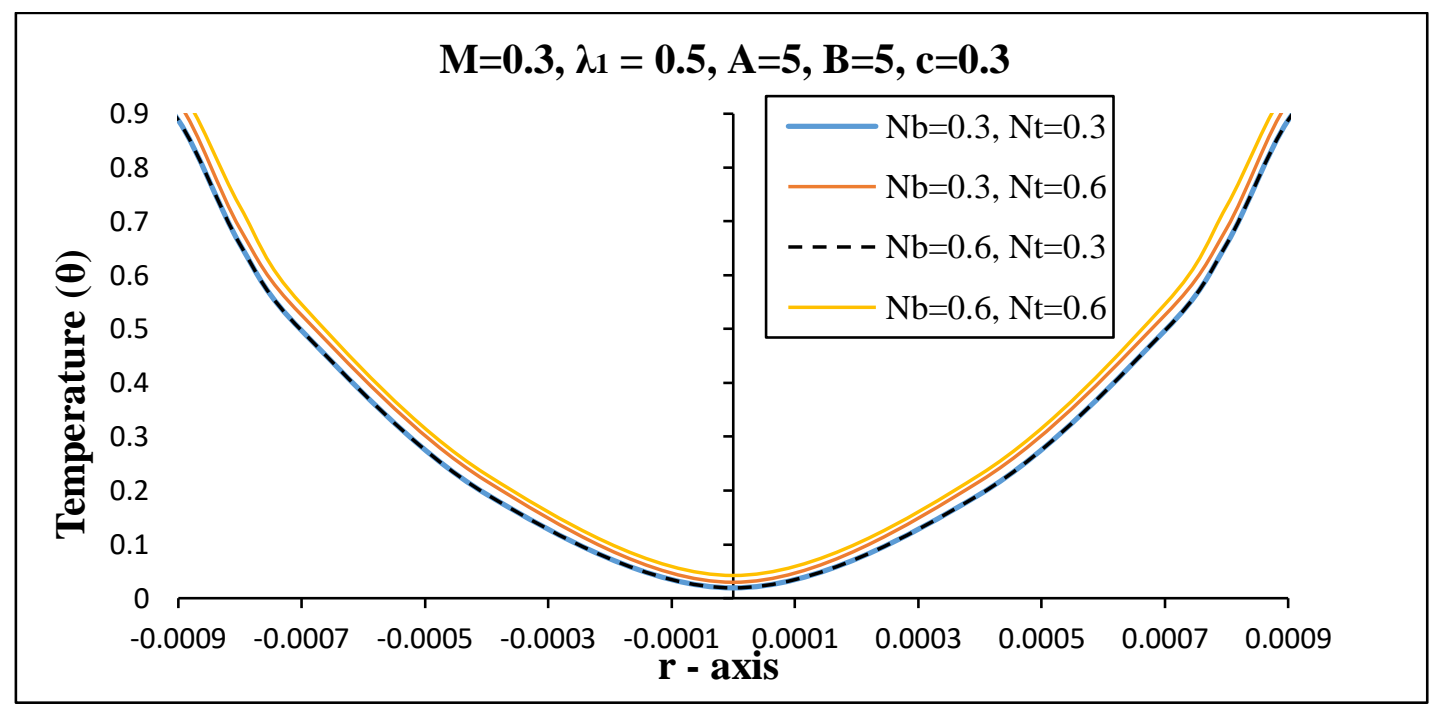

Figure 19 Temperature profile for diameter of stenosis $=0.4 l$ at $z=0.005$

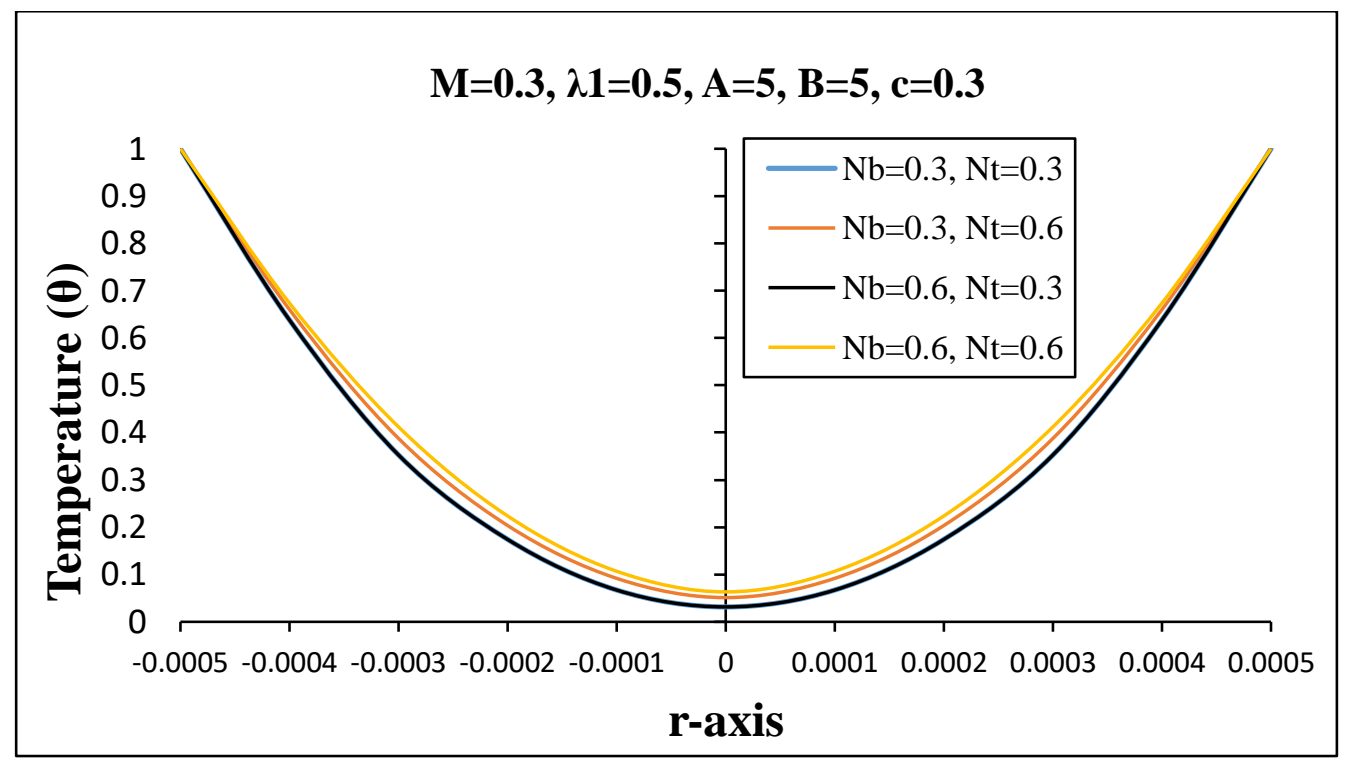

Figure 20 Temperature profile for diameter of stenosis $=0.3 l$ at $z=0.005$ 


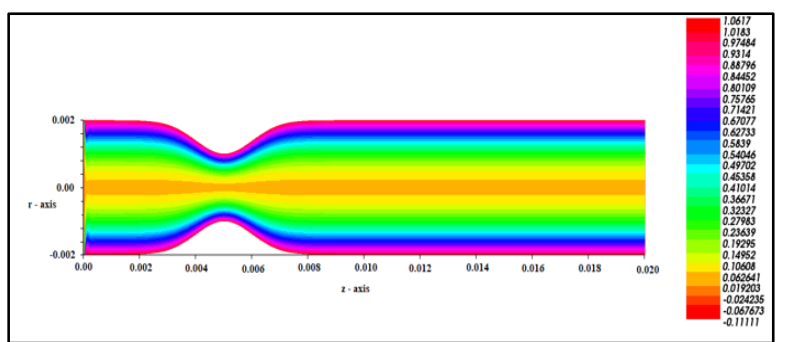

21(a)

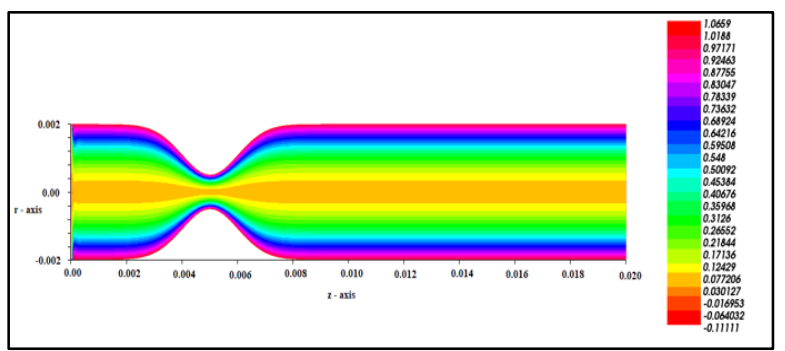

21(c)

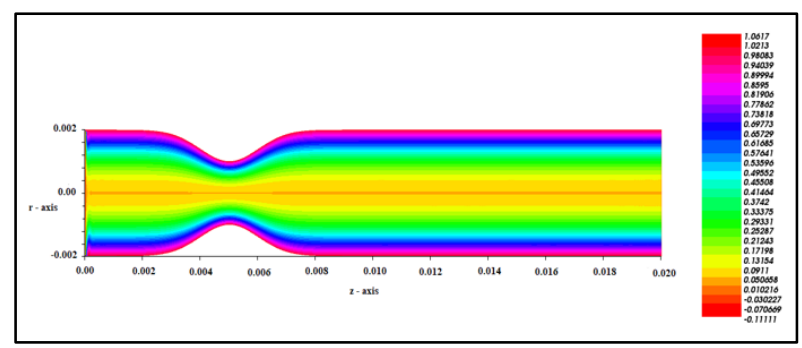

21(b)

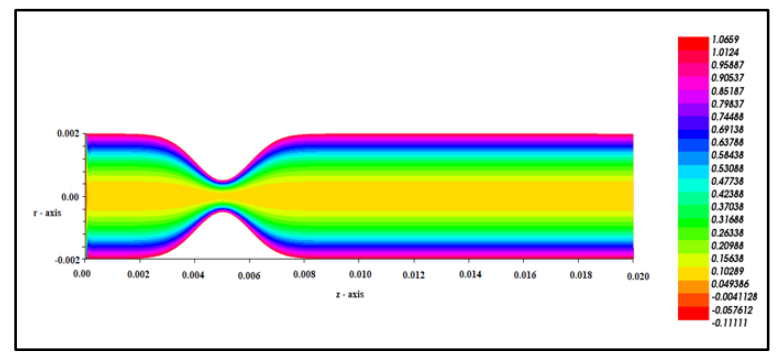

21(d)

Figure 21 Temperature distribution (a) $\lambda_{1}=-0.5, d=0.4 l, N_{b}=0.3$ and $N_{t}=0.3$, (b)

$\lambda_{1}=-0.5, d=0.4 l, N_{b}=0.3$ and $N_{t}=0.6,(c) \lambda_{1}=-0.5, d=0.3 l, N_{b}=0.3$ and $N_{t}=0.3$, (d) $\lambda_{1}=-0.5, d=0.3 l, N_{b}=0.3$ and $N_{t}=0.6$

Figure 21 illustrates the temperature contour plots with various values of stenotic diameter $(d)$ and thermophoresis parameter $\left(N_{t}\right)$ along the arterial domain for a fixed value of Brownian motion parameter $\left(N_{b}=0.3\right)$ and viscoelastic parameter $\left(\lambda_{1}=-0.5\right)$. From fig. $21 \mathrm{a}$ to $21 \mathrm{~b}$ the thermophoresis parameter $N_{t}$ is increased from 0.3 to 0.6 and both graphs correspond to the wider stenotic region $(d=0.4 l)$. This generates an expansion in the hotter zone to an around the mid-line of the domain i.e. darker orange contours are replaced by lighter ones. Temperature in the vicinity of the constriction is therefore elevated both axially and radially and this concurs with earlier graphs. This trend is also observed by comparing figs. $21 \mathrm{c}$ and $21 \mathrm{~d}$ which relate to the narrower stenotic region $(d=0.3 l)$ and again correspond to the same increase in thermophoresis parameter. Comparing fig. 21a with 21c (which corresponds to a reduction in stenotic diameter from $d=0.4 l$ to $0.3 l$ ), the yellow/orange central zone computed in fig. $21 \mathrm{a}$ is considerably expanded around the mid-line indicating that a narrower geometry reduces thermal transport along the axis of the artery and decreases temperatures in the blood flow. The yellow contours correspond to higher temperatures and these are constricted while the cooler orange contours are expanded. This trend is sustained before and after the stenotic region along the entire axial length of the arterial section. There is no significant modification in temperature contours in the vicinity of the arterial walls (upper and lower boundaries) in any of the plots i.e. no tangible alteration in temperature near the walls is caused by a change in stenotic diameter or thermophoresis parameter. 
Figure 22 shows the radial temperature distribution in the arterial stenosed blood flow with variation of thermophoresis parameter $\left(N_{t}\right)$ and Brownian motion parameter $\left(N_{b}\right)$ for magnetic parameter, $M=0.6$ for the wider (first) case of stenosis $(d=0.4 l)$. With increasing thermophoresis body force (i.e. larger $N_{t}$ ) there is a notable elevation in temperatures across the arterial crosssection i.e. with all values of radial coordinate. A strong plateau is computed in the central midline zone of the arterial vessel. Maximum temperatures correspond to the walls of the vessel and the minimum values arise in the centre of the vessel. With increasing Brownian motion parameter temperatures are found to be markedly decreased in the central zone (plateau region around the mid-line) whereas they are weakly enhanced in the peripheral regions around the core zone. Cooling of the blood flow around the central core region is therefore induced with greater Brownian motion i.e. smaller sized nano-particles.

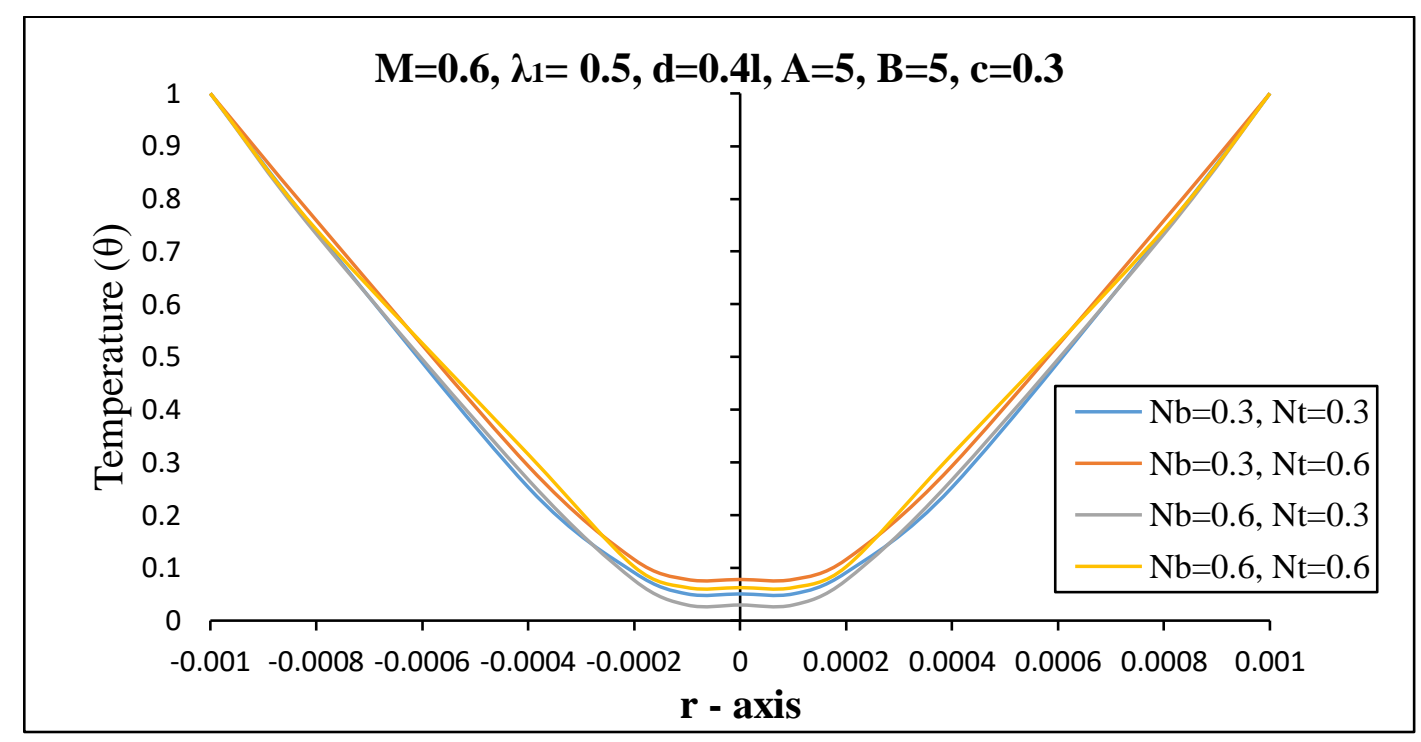

Figure 22 Temperature profile for diameter of stenosis $d=0.4 l$ at, $z=0.005$

Figure 23 shows the radial temperature distribution with different values of magnetic body force parameter $(M)$ and also various thermophoresis values $\left(N_{t}\right)$ for the wider stenotic case. A distinct elevation in temperature is computed across the vessel cross-section. The supplementary work expended in dragging the blood against the action of the radial magnetic field is dissipated as heat. This excess heat energizes the blood flow and manifests in temperature elevation. The associated thermal boundary layer thickness at the vessel walls will also be increased. This confirms the findings of many investigators in magnetohydrodynamics including Cramer and Pai [70]. Static magnetic fields may therefore prove very beneficial in thermal treatment of blood flow disorders as noted by Brix et al. [71]. Again it is also apparent that higher values of nanoscale thermophoresis 
parameter $\left(N_{t}\right)$ induces a clear enhancement in temperatures for all radial locations of the arterial vessel, although the principal elevation is observed around the central zone and diminishes progressively towards the vessel boundaries.

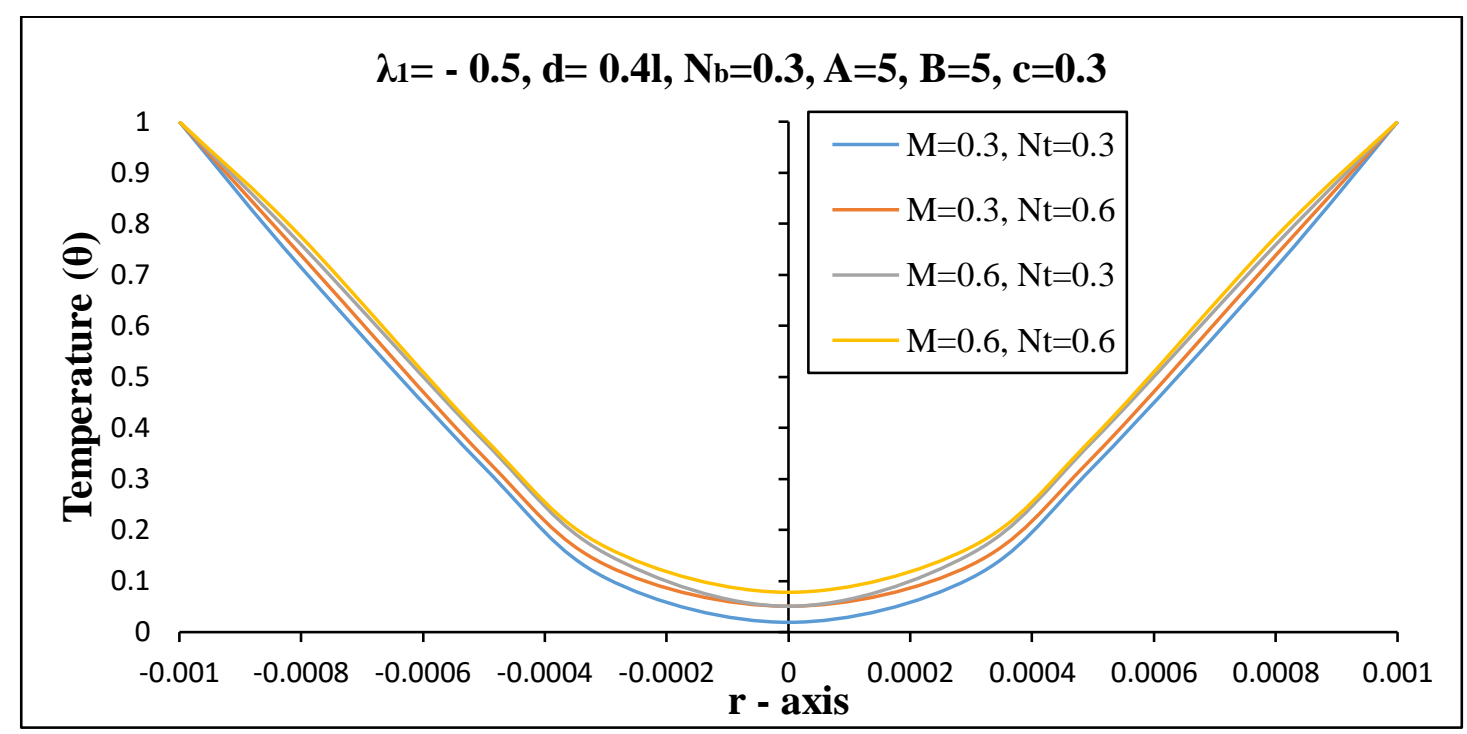

Figure 23 Temperature profile for diameter of stenosis $=0.4 l$ at, $z=0.005$

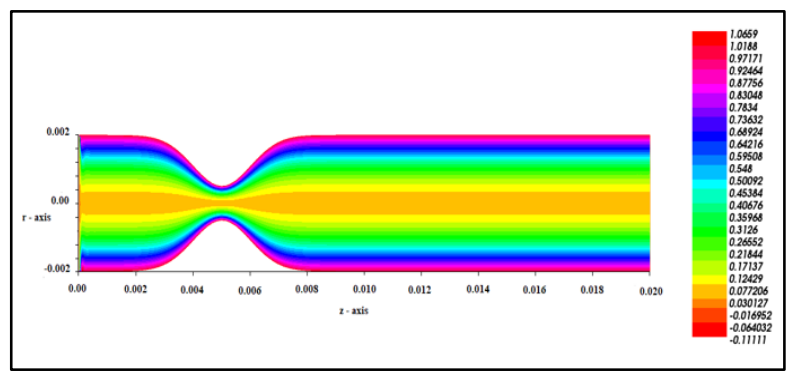

24(a)

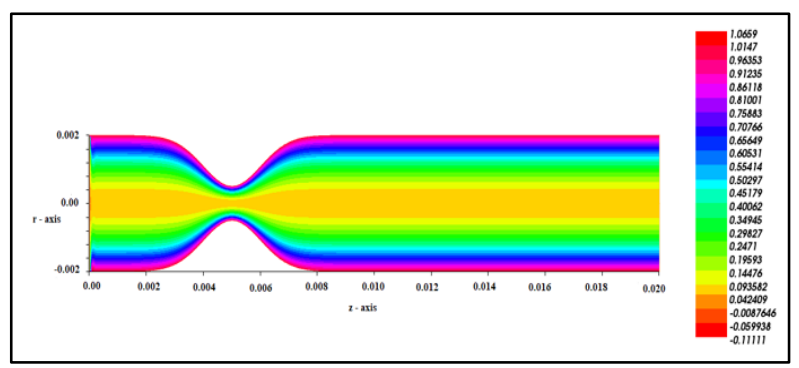

24c)

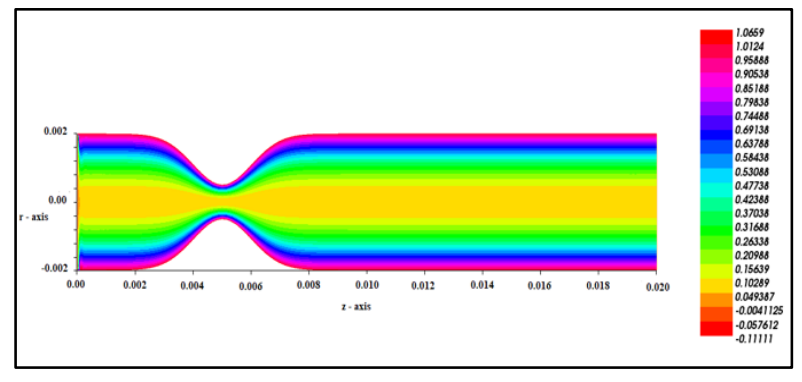

24(b)

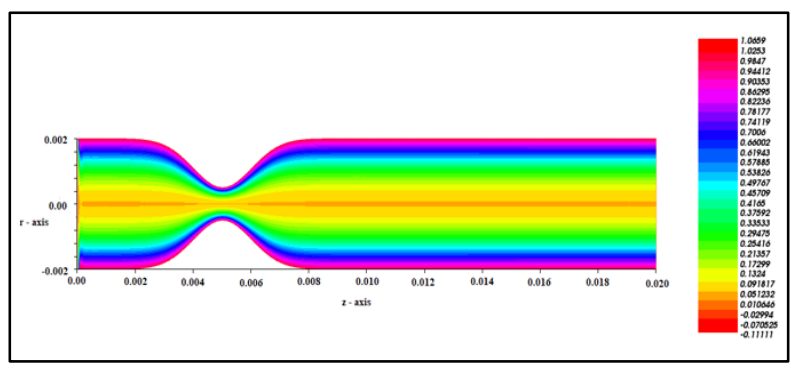

24(d)

Figure 24 Nanoparticle Concentration for (a) $\lambda_{1}=-0.5, M=0.3, N_{b}=0.3$ and $N_{t}=0.3$, (b)

$\lambda_{1}=-0.5, M=0.3, N_{b}=0.3$ and $N_{t}=0.6$, (c) $\lambda_{1}=-0.5, M=0.6, N_{b}=0.3$ and $N_{t}=0.3$ (d) $\lambda_{1}=-0.5, M=0.6, N_{b}=0.3$ and $N_{t}=0.6$.

Figures 24(a) - (d) visualize the nanoparticle concentration contours for various magnetic body force parameters $(M)$ and thermophoresis parameters $\left(N_{t}\right)$ with fixed values of Brownian motion parameter $\left(N_{b}=0.3\right)$ and viscoelastic parameter $\left(\lambda_{1}=-0.5\right)$. Comparing fig. $24 \mathrm{a}$ with fig $24 \mathrm{~d}$ the 
thermophoresis parameter is increased from 0.3 to 0.6 with all other parameters constrained, there is an expansion in the orange (lower magnitude contour) zone is expanded and the engulfing yellow zone (higher magnitude contour) is contracted. The values for nano-particle concentration are therefore reduced along the axial direction and radial direction in the vicinity of the stenotic region. Species diffusion of nano-particles into the core region is therefore decreased with greater thermophoretic effect. Comparing fig. 24a with fig. 24c, the magnetic parameter is increased from $M=0.3$ to 0.6 , with all other parameters fixed. A similar response to the thermophoretic effect is observed i.e. the orange zone is expanded and the yellow zone is diminished Stronger magnetic field therefore also inhibits nano-particle diffusion into the core zone and results in decreasing magnitudes of nano-particle concentration along the entire arterial section i.e. with all axial coordinate locations. Comparing fig. $24 \mathrm{c}$ and $24 \mathrm{~d}$ this trend is further amplified with the emergence of a thin brown zone along the arterial mid-line indicating an even greater depletion in nano-particle concentration values. The combination of maximum magnetic parameter value and maximum thermophoresis parameter value therefore serves to strongly diminish nano-particle concentration values. The opposite effect i.e. elevation in nano-particle diffusion may therefore be induced by utilizing a weaker magnetic field and lower thermophoresis in nano-particle deployment in stenotic blood flows. Generally, no significant modification in nano-particle concentration distribution at the vessel walls is induced with a change in either magnetic body force parameters $(M)$ and thermophoresis parameters $\left(N_{t}\right)$.

Fig. 25 illustrates the influence of Brownian motion parameter $\left(N_{b}\right)$ and thermophoresis parameter $\left(N_{t}\right)$ on radial distribution of the nano-particle concentration $(\phi)$ at low magnetic field $(M=0.3)$ and for the wider stenosis case $(d=0.4 l)$. Parabolic profiles are computed across the vessel crosssection with maximum nano-particle concentrations at the vessel walls and the minimum values clustered around the arterial mid-line. With increasing thermophoresis parameter there is a strong boost in magnitudes of nano-particle concentration for all radial coordinates, as opposed to the axial decrease computed earlier in figs. 24a-d. Similarly, there is a marked elevation in nanoparticle concentrations with increasing Brownian motion parameter from 0.3 to 0.6. Both nanoscale effects therefore assist in the diffusion of the nano-particle species at all radial coordinates although the effect is maximized at intermediate distances from the arterial mid-line. Brownian motion acts to distribute the nano-particles as uniformly as possible throughout the blood flow regime. This reduces the nanoparticle concentration gradient and diminishes the regional variations in fluid characteristics whereas it encourages species diffusion leading to an elevation in nano-particle concentration magnitudes. 


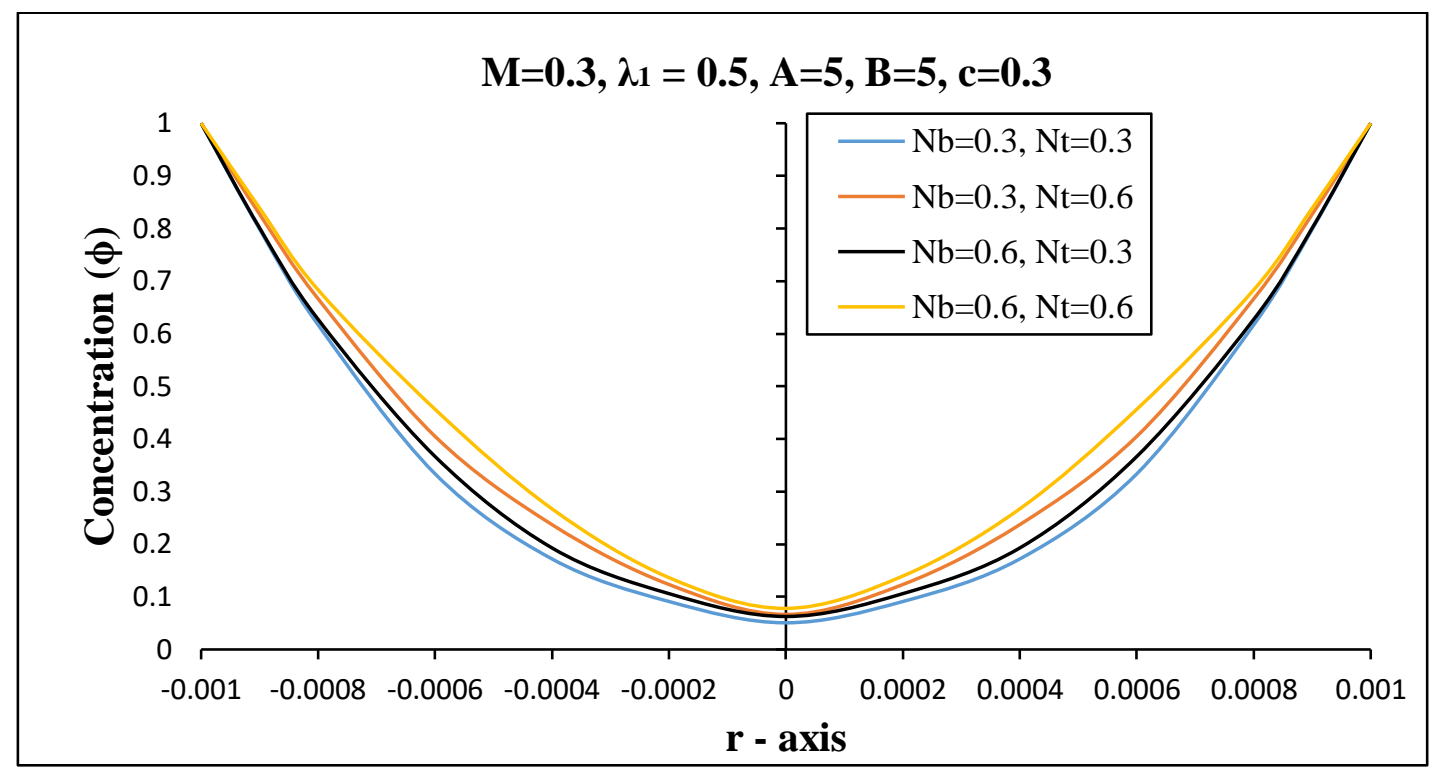

Figure 25 Nanoparticles concentration for diameter of stenosis $=0.4 l$ at $z=0.005$

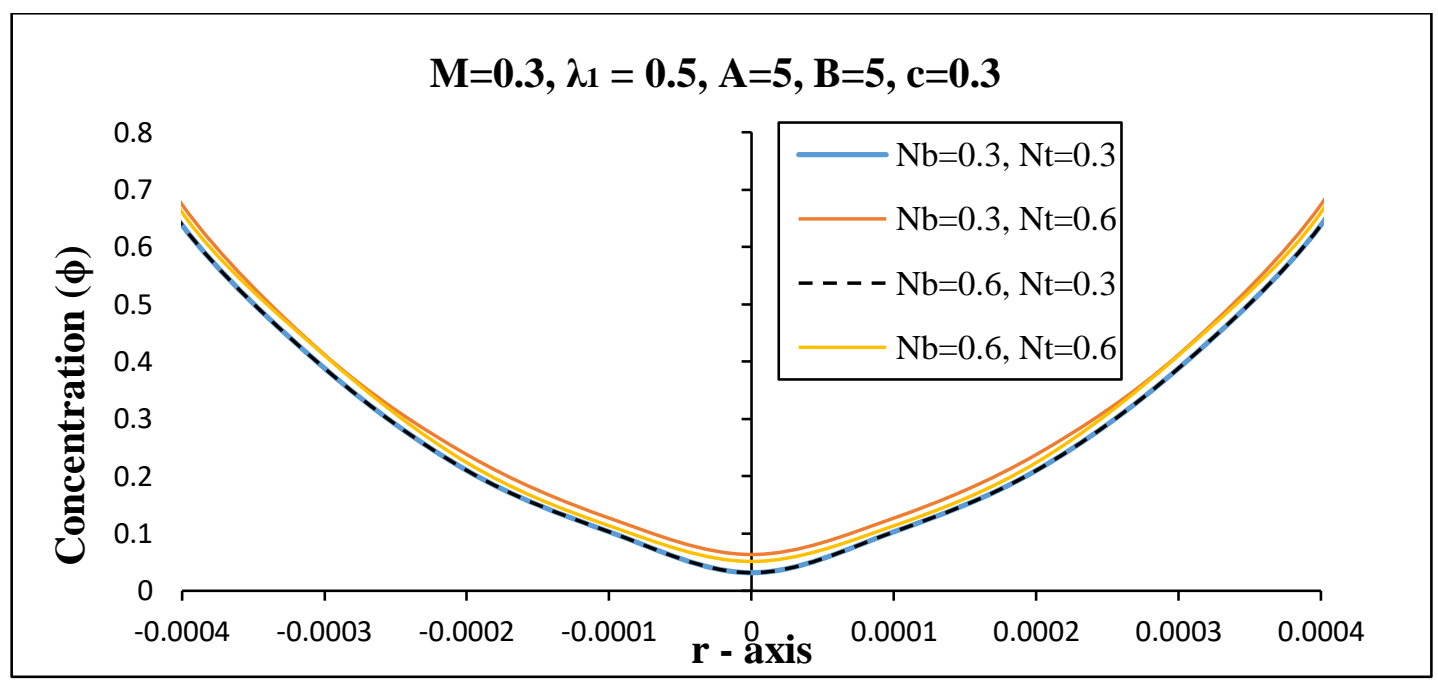

Figure 26 Nanoparticles concentration for diameter of stenosis $=0.3 \mathrm{l}$ at $z=0.005$

Fig. 26 depicts the impact of Brownian motion $\left(N_{b}\right)$ and thermophoresis parameter $\left(N_{t}\right)$ for the narrower stenosis case $(d=0.3 l)$. Comparing Fig. 26 (narrow stenosis) with fig. 25 (wider stenosis) over the range $-0.0004<r<0.0004$ it is immediately evident that much higher magnitudes of nano-particle concentration are obtained across the vessel cross-section i.e. along the radial direction. The narrower stenosis therefore assists in the diffusion of nano-particles through the blood flow across the vessel cross-section. Parabolic distributions are again obtained for all combinations of $N_{b}$ and $N_{t}$. Again, elevation in $N_{t}$ value leads to accentuation in the nano-particle concentration values. However, an increase in $N_{b}$ values, in this narrower stenotic case, manifests with a weak reduction in the nano-particle concentration values. 


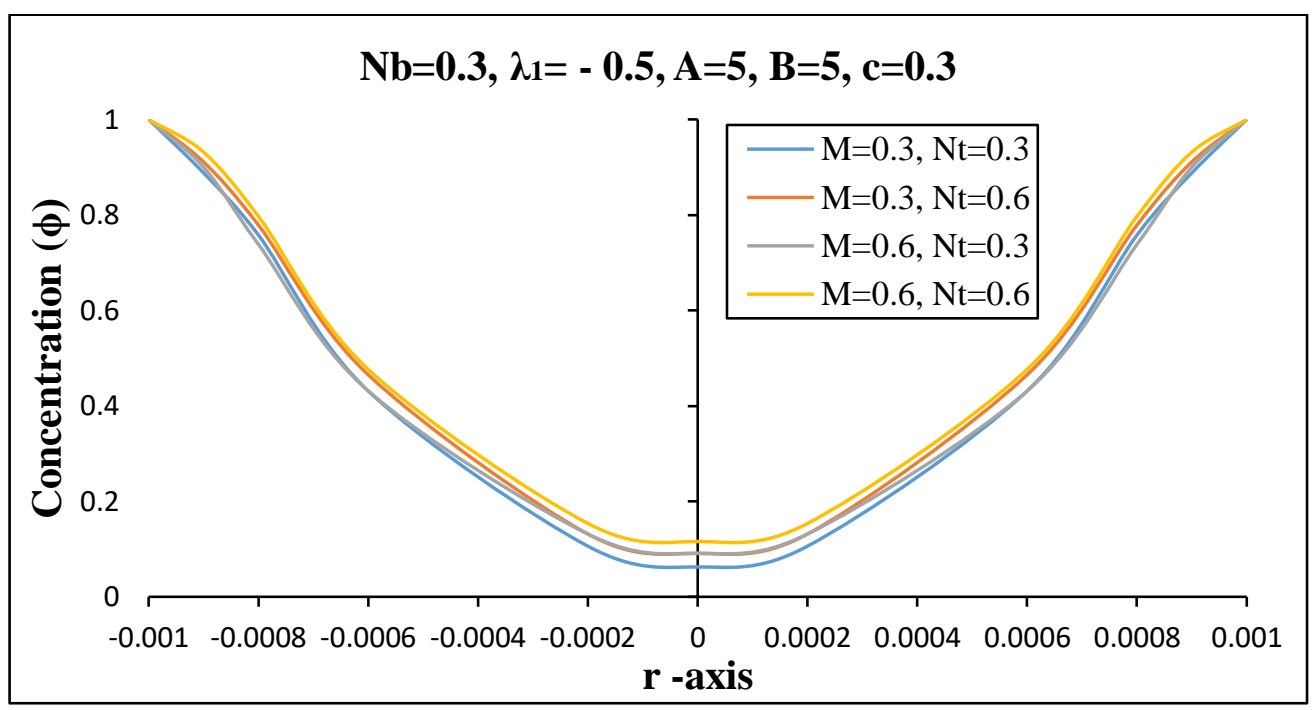

Figure 27 Nanoparticles concentration for diameter of stenosis $=0.4 l$ at $z=0.005$

Fig. 27 shows the variation of nano-particle concentration with magnetic parameter $(M)$ and thermophoresis parameter $\left(N_{t}\right)$. An increment in thermophoresis parameter generally enhances magnitudes of the nano-particle concentration for at all radial locations. Similarly, nano-particle concentration magnitudes are also elevated with greater $M$ value i.e. stronger radial magnetic field encourages nano-particle species diffusion in the blood flow.

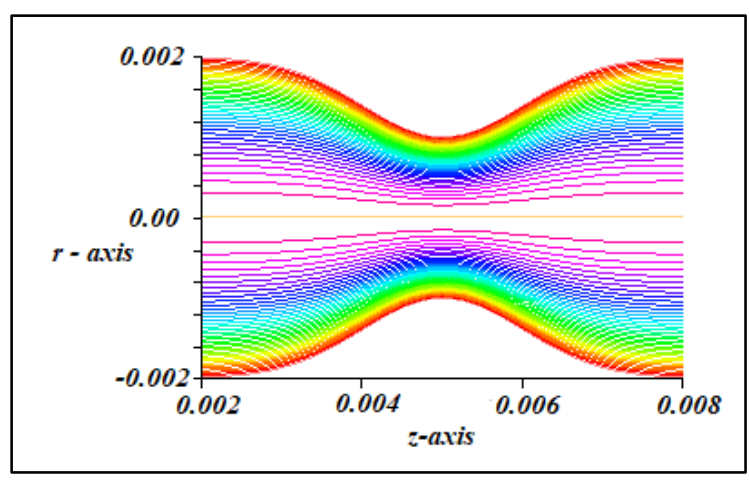

28(a)

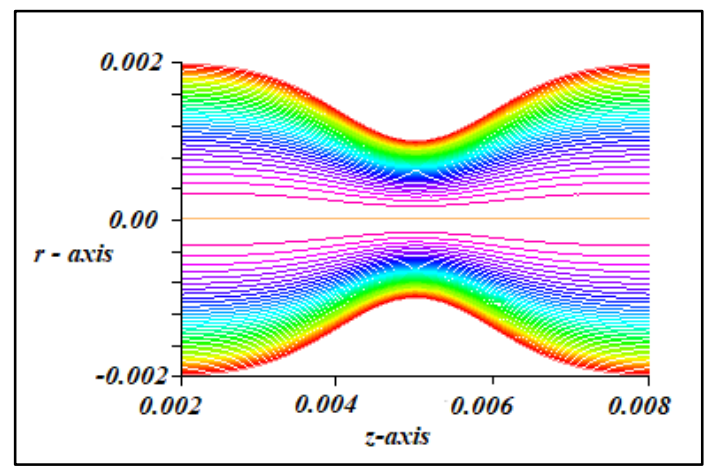

28(b)

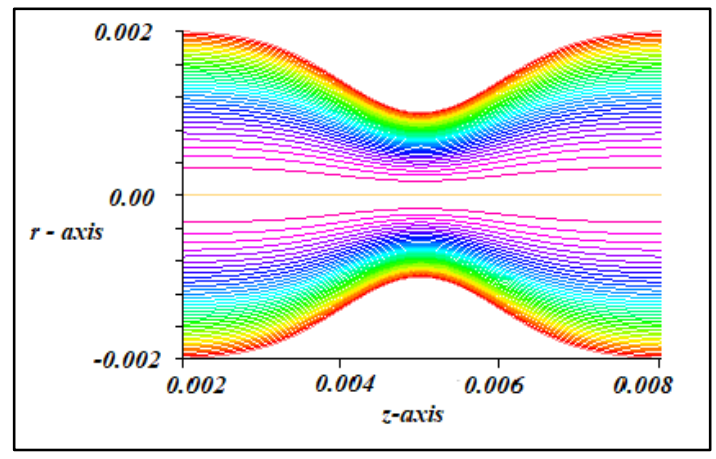

28(c)

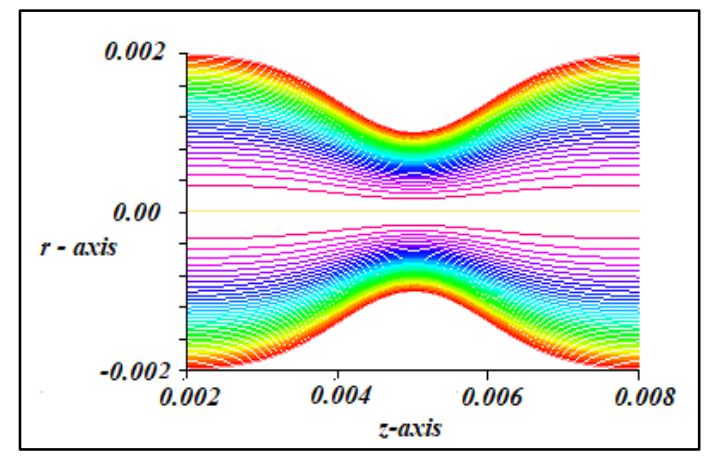

28(d) 
Figure 28 Streamlines of blood flow in the arterial segment when a) $M=0.3, N_{t}=0.3$

b) $M=0.3, N_{t}=0.6$ c) $M=0.6, N_{t}=0.3$ d) $M=0.6, N_{t}=0.6$

Finally, Figs. 28 (a) to (d) depict the streamlines of blood flow for specific values of $M$ and $N_{t}$ when $N_{b}=0.3, \lambda_{1}=0.5, A=B=5$ and $c=0.3$. In Fig 28(a), the presence of a circulating bolus of blood enclosed by the streamlines in the stenotic region of the artery represents that if the size of the bolus will be decreased, the flow acceleration will also be decreased at the stenotic part of the artery. The bigger the bolus the higher the acceleration at that point. It can be seen from the two figures 28(a) and 28(b), by applied magnetic field the strength of the circulating region reduced. But comparing the 28(a) with 28(c), even with alteration in magnetic parameter $(M)$ there seems no significant deviation in size and circulation of this bolus of blood. This indicates that the circulating bolus of blood is relatively insensitive to magnetic field. However, inspection of Figs. 28(b), 28(d), shows that there is some deviation in the characteristics (magnitude and structure) of the bolus of blood with a change in thermophoresis parameter. It is further of note that the circulating bolus in is a trapped vortex zone generated in internal hemodynamics. It is an important flow characteristic which signifies a modification in flow deceleration/acceleration at the stenotic part of the artery. It has been popularized for a number of decades in hemodynamics simulations, notably by Fung [72] and also Yoganathan et al. [73] and Zien et al. [74]. In consistency with these studies, our simulations show that the larger the bolus the higher the acceleration at that point. A good perspective of the internal flow dynamics in blood behaviour is therefore furnished with bolus dynamic consideration. Of course, this may be further analysed with three-dimensional simulations in the future.

\section{CONCLUSIONS}

In the present paper, motivated by providing a deeper understanding of nano-drug diffusion in the treatment of cardiovascular disease (stenotic arteries), a finite element simulation of twodimensional magnetohydrodynamic viscoelastic blood flow doped with nano-particles in a stenosed arterial geometry has been presented. The Reiner-Rivlin second order differential model has been adopted to mimic non-Newtonian effects and the Buongiorno nanoscale model employed to analyse Brownian motion and thermophoresis effects. The non-dimensional conservation equations for momentum, heat and nano-particle species with appropriate boundary conditions are 
solved numerically by means of the finite element method based on the variational approach and simulated using the FreeFEM++ code. Modification in hemodynamic velocity, heat and mass transfer characteristics are studied for the effects of geometric, nanoscale, rheological, viscosity and magnetic parameters i.e. viscoelastic parameter $\left(\lambda_{1}\right)$, thermophoresis parameter $\left(N_{t}\right)$, Brownian motion parameter $\left(N_{b}\right)$ and magnetic body force parameter $(M)$ at the throat of the stenosis and the domain. The main outcomes from the present investigation are as follows:

- It is observed that in the first stenosis case (i.e., wider stenosis diameter, $d=0.41$ ), increasing the thermophoresis parameter $\left(N_{t}\right)$ generates an increase in nanoparticles concentration and temperature values in the regime.

- For the wider stenosis case, increasing thermophoresis parameter and magnetic body force parameter both result in an elevation in temperature and nanoparticle concentration whereas they strongly reduce velocity (i.e. decelerate the blood flow) both at the stenosis and throughout the whole domain.

- For the second stenosis case (i.e. narrower stenosis diameter $d=0.3 l$ ) there is a deceleration in the flow in the vicinity of the stenotic region. However, there is a marked acceleration in the blood flow with increasing Brownian motion parameter $\left(N_{b}\right)$ in the entire arterial geometry simulated.

- For the second stenosis case, there is a generally an elevation in temperature and nanoparticle concentration with higher values of magnetic parameter $(M)$ and thermophoresis parameter $\left(N_{t}\right)$.

- The skin-friction coefficient is elevated significantly for the narrower stenosis geometry (second case, $d=0.3 l$ ).

- For higher values of magnetic body force (MHD) parameter $M(M \geq 1)$, the values of skin friction coefficient increase rapidly in comparison with lower values of $M$.

- For the larger stenosis geometry (first case, $d=0.4 l$ ) there is a sustained linear growth in skin friction with radial coordinate whereas for the narrower stenosis (second case, $d=0.3 l$ ) there is a variation in the gradient.

- An increase in the Vogel viscosity model parameter, $A$ decelerates the blood flow whereas the converse effect i.e. enhancement in velocity is induced with increasing Vogel parameter, $c$.

- Due to viscosity being taken as a function of temperature (which provides a better approximation for actual cardiovascular hemodynamics based on clinical observations), the velocity decreases at the walls of the artery as compared to the case where viscosity is taken as 
a constant. Furthermore, due to increased drag at the walls, the skin friction coefficient is increased which relates to a transition in the flow from laminar towards turbulent flow and this case is much closer to the actual case of blood flow in a stenotic artery. Therefore, the simulations provide an improved insight into diagnosing the cardiovascular disease formation, modelling of drugs through the diseased arteries and testing the designs of heart valves, stents etc.

- An increase in positive viscoelastic parameter $\left(\lambda_{1}\right)$ generates much higher skin friction magnitudes due to the elevation in viscosity of the rheological blood. The reverse behaviour is computed with negative viscoelastic parameter $\left(\lambda_{1}\right)$ values.

- Bolus structure and magnitude exhibits a greater sensitivity to thermophoresis nanoscale effect than to magnetic field. This indicates that nanoparticles may provide a more useful mechanism for treatment than extra-corporeal magnetic fields in cardiovascular diagnostics and clinical treatments.

The present study has been confined to two-dimensional geometric configurations and has produced interesting computations with the FreeFEM++ code. Future investigations will consider more complex three-dimensional geometries [75] and also alternative non-Newtonian models e.g. the Oldroyd-B viscoelastic model.

\section{Acknowledgements}

The authors are grateful to Science and Engineering Research Board (SERB), Department of Science and Technology (DST), Govt of India for undertaking the research work under the research project File Number: ECR/2017/001053 dated 12/03/2018. Furthermore, all the authors wish to express their gratitude to the reviewers for their excellent comments which have served to improve the present work.

\section{REFERENCES}

[1]. Young F D., Tsai F.Y., Flow characteristics in model of arterial stenosis steady flow, $J$. Biomech. 6:395-410, 1973.

[2]. Ku D.N., Blood flow in arteries, Ann. Rev. Fluid Mech., 29:399-434, 1997

[3]. Fung, Y.C., Biomechanics: Mechanical Properties of Living Tissues, Springer-Verlag, New York, 1981. 
[4]. Merrill, Edward W. Rheology of human blood and some speculations on its role in vascular homeostasis. Biomechanical Mechanisms in Vascular Homeostasis and Intravascular Thrombus, New York, USA, 1965.

[5]. Taylor, M. G., The influence of the anomalous viscosity of blood upon its oscillatory flow. Physics in Medicine \& Biology, 3, no. 3: 273, 1959.

[6]. Baieth, HE Abdel, Physical parameters of blood as a non-Newtonian fluid. International Journal of Biomedical Science: IJBS 4, no. 4: 323, 2008.

[7]. Thiriet M., Biomathematical and Biomechanical Modelling of the Circulatory and Ventilatory Systems. Vol 2: Control of Cell Fate in the Circulatory and Ventilatory Systems. Springer Math\& Biological Modeling, New York, USA, 2011.

[8]. Mekheimer, K.S. and El Kot, M.A., Mathematical modelling of unsteady flow of a Sisko fluid through an anisotropically tapered elastic arteries with time-variant overlapping stenosis. Applied Mathematical Modelling, 36(11), 5393-5407, 2012.

[9]. Riahi, D. N., Roy, R. and Cavazos, S., On arterial blood flow in the presence of an overlapping stenosis. Mathematical and Computer Modelling. 54. 2999-3006, 2011.

[10]. Ellahi, R., Rahman, S. U, Gulzar, M., Nadeem, S., and Vafai, K., A mathematical study of non-Newtonian micropolar fluid in arterial blood flow through composite stenosis, Applied Mathematics \& Information Sciences. 4. 1567-1573, 2014.

[11]. Tzirakisa, K. Botti, L. Vavourakis, V. and Papaharilaou, Y., Numerical modelling of nonNewtonian biomagnetic fluid flow, Computers and Fluids 126. 170-180, 2016.

[12]. Akbar, Noreen Sher, Nadeem S., and Mohamed Ali., Jeffrey fluid model for blood flow through a tapered artery with a stenosis. Journal of Mechanics in Medicine and Biology 11, no. 03: 529-545, 2011.

[13]. Chakravarty, Santabrata, and Mandal Prashanta Kumar, Two-dimensional blood flow through tapered arteries under stenotic conditions. International Journal of Non-Linear Mechanics, 35, no. 5: 779-793, 2000.

[14]. Moreno, Carlos, and Bhaganagar Kiran, Modeling of Stenotic coronary artery and implications of plaque morphology on blood flow, Modelling and Simulation in Engineering 2013: 14, 2013.

[15]. Haghighi, A. R., \& Chalak, S. A., Mathematical modelling of blood flow through a stenosed artery under body acceleration. Journal of the Brazilian Society of Mechanical Sciences and Engineering, 39(7), 2487-2494, 2017.

[16]. Tripathi, D., A mathematical study on three layered oscillatory blood flow through stenosed arteries, Journal of Bionic Engineering. 9. 119-131, 2012. 
[17]. Mishra, S., Siddiqui S.U. and Medhavi, A., Blood flow through a composite stenosis in an artery with permeable wall, Applications and Applied Mathematics. 6(1). 1798-1813, 2011.

[18]. Nadeem, S., and Akbar Noreen Sher, Simulation of the second-grade fluid model for blood flow through a tapered artery with a stenosis, Chinese Physics Letters 27, no. 6: 068701, 2010.

[19]. Ramesh, K., and Devakar, M., The effects of endoscope and heat transfer on the peristaltic flow of a second-grade fluid in an inclined tube, Journal of Mechanics in Medicine and Biology, 16(04), 1650057, 2016.

[20]. Srivastav, R. K., Mathematical model of blood flow through a composite stenosis in catheterized artery with permeable wall, Applications and Applied Mathematics. 99. 58-74, 2014.

[21]. Jayaraman, G. and Tewari, K., Flow in catheterized curved artery, Medical \& Biological Engineering \& Computing. 33. 720-724, 1995.

[22]. Srivastava, V. P., and Srivastava, R., Particulate suspension blood flow through a narrow catheterized artery, Computers \& Mathematics with Applications. 58. 227-238, 2009.

[23]. Yousef Haik, Vinay Pai, and Ching-Jen Chen. Apparent viscosity of human blood in a high static magnetic field, Journal of Magnetism and Magnetic Materials, 225(1-2):180-186, 2001.

[24]. Yadav R. P, Harminder S., and Bhoopal S., Experimental studies on blood flow in stenosis arteries in presence of magnetic field, Ultra Sci, 20(3):499-504, 2008.

[25]. Nadeem, S., Ijaz, S. and Sadiq, M.A., Inspiration of induced magnetic field on a blood flow of Prandtl nanofluid model with stenosis, Current Nanoscience. 13. 753-765, 2014.

[26]. Kh S Mekheimer, Effect of the induced magnetic field on peristaltic flow of a couple stress fluid, Physics Letters A, 372(23):4271-4278, 2008.

[27]. Kh S Mekheimer and MA El Kot, Influence of magnetic field and hall currents on blood flow through a stenotic artery. Applied Mathematics and Mechanics, 29(8):1093, 2008.

[28]. Silvio Cavalcanti. Hemodynamics of an artery with mild stenosis. J. Biomechanics, 28(4):387-399, 1995.

[29]. Tzirtzilakis, E. E., A mathematical model for blood flow in magnetic field, Physics of Fluids, 17, no. pp. 7-15, 2005.

[30]. Ponalagusamy R. and Tamil Selvi R., Influence of magnetic field and heat transfer on twophase fluid model for oscillatory blood flow in an arterial stenosis, Meccanica, 50(4):927943,2015 
[31]. Ponalagusamy R. and Priyadharshini S., Numerical investigation on two-fluid model (micropolar-Newtonian) for pulsatile flow of blood in a tapered arterial stenosis with radially variable magnetic field and core fluid viscosity, Computational and Applied Mathematics, 37, 719-743, 2018.

[32]. Choi, S. U. S., and J. A. Eastman, Enhancing thermal conductivity of fluids with nanoparticles, ASME-Publications-Fed 231: 99-106, 1995.

[33]. Buongiorno, Jacopo, Convective transport in nanofluids. ASME J. Heat Transfer, 128, 3: 240-250, 2006.

[34]. Masuda, Hidetoshi, Akira Ebata, and Kazumari Teramae., Alteration of thermal conductivity and viscosity of liquid by dispersing ultra-fine particles. Dispersion of $\mathrm{Al}_{2} \mathrm{O}_{3}, \mathrm{SiO}_{2}$ and $\mathrm{TiO}_{2}$ ultra-fine particles, Netsu Bussei, 7, 227-233, 1993.

[35]. Wong K. F. V., Bon B. N.,Vu S., and Samedi S., Study of nanofluid natural convection phenomena in rectangular enclosures, ASME International Mechanical Engineering Congress and Exposition (IMECE '07), vol. 6, pp. 3-13, Seattle, Wash, USA, November, 2007.

[36]. Xuan, Yimin, and Wilfried Roetzel. Conceptions for heat transfer correlation of nanofluids. International Journal of heat and Mass transfer 43, no. 19: 3701-3707, 2000.

[37]. Xuan, Yimin, and Qiang Li., Investigation on convective heat transfer and flow features of nanofluids. ASME J. Heat Transfer, 125, no. 1: 151-155, 2003.

[38]. Giljohann, David A., Dwight S. Seferos, Weston L. Daniel, Matthew D. Massich, Pinal C. Patel, and Chad A. Mirkin., Gold nanoparticles for biology and medicine. Angewandte Chemie International Edition 49, no. 19: 3280-3294, 2010.

[39]. Kumar, K. Praveen, Willi Paul, and Chandra P. Sharma., Green synthesis of gold nanoparticles with Zingiber officinale extract: characterization and blood compatibility, Process Biochemistry 46, no. 10: 2007-2013, 2011.

[40]. Bég, O. Anwar, Prasad, V. R., and Vasu, B., Numerical study of mixed bioconvection in porous media saturated with nanofluid containing oxytactic microorganisms. Journal of Mechanics in Medicine and Biology, 13(04), 1350067, 2013.

[41]. Ali, Nasir, Zaman A., Sajid M., Bég, Anwar O., Shamshuddin M. D. and Ali Kadir., Numerical simulation of time-dependent non-Newtonian nanopharmacodynamic transport phenomena in a tapered overlapping stenosed artery, Nanoscience and Technology: An International Journal, 9, 247-282, 2018.

[42]. H. Vogel, Die Bedeutung der Temperatur Abhangigkeit der Viskositat f. d. Beurteilung von Oelen, Z Angew. Chem. 35 (1922) 561. 
[43]. Pakdemirli, M., and Yilbas B. S., Entropy generation for pipe flow of a third-grade fluid with Vogel model viscosity, International Journal of Non-Linear Mechanics 41, no. 3: 432-437, 2006.

[44]. Ellahi, Rahmat, Raza M., and Vafai K., Series solutions of non-Newtonian nanofluids with Reynolds' model and Vogel's model by means of the homotopy analysis method, Mathematical and Computer Modelling 55, no. 7-8: 1876-1891, 2012.

[45]. Akbar, Noreen Sher, Nadeem S., Hayat T., and Hendi Awatif A., Analytical and numerical analysis of Vogel's model of viscosity on the peristaltic flow of Jeffrey fluid, ASCE J. Aerospace Engineering 25, no. 1: 64-70, 2011.

[46]. Malik, M. Y., Hussain A., and S. Nadeem. Boundary layer flow of an Eyring-Powell model fluid due to a stretching cylinder with variable viscosity, Scientia Iranica 20, no. 2: 313-321, 2013.

[47]. Hatami, M., Hatami J., and Ganji Davood Domiri. Computer simulation of MHD blood conveying gold nanoparticles as a third grade non-Newtonian nanofluid in a hollow porous vessel, Computer Methods Programs Biomedicine 113, no. 2: 632-641, 2014.

[48]. Akinshilo, Akinbowale T., and Osamudiamen Olaye. On the analysis of the Erying Powell model based fluid flow in a pipe with temperature dependent viscosity and internal heat generation. Journal of King Saud University-Engineering Sciences(2017). https://doi.org/10.1016/i.jksues.2017.09.001

[49]. 49.Sheikholeslami, M., Gorji-Bandpy M., and Soleimani Soheil, Two-phase simulation of nanofluid flow and heat transfer using heatline analysis, International Communications in Heat and Mass Transfer 47: 73-81: 2013.

[50]. Aziz, Asim, and Aziz Taha, MHD flow of a third-grade fluid in a porous half space with plate suction or injection: an analytical approach, Applied Mathematics and Computation, 218, no. 21: 10443-10453, 2012.

[51]. Sajid, M., Ali, N., Bég, O. Anwar, \& Siddiqui, A. M., Swimming of a singly flagellated microorganism in a magnetohydrodynamic second-order fluid. Journal of Mechanics in Medicine and Biology, 17(01), 1750009, 2017.

[52]. Dunn, Ernest J., and Fosdick Roger L., Thermodynamics, stability, and boundedness of fluids of complexity 2 and fluids of second grade, Archive for Rational Mechanics and Analysis 56, no. 3: 191-252, 1974. 
[53]. Dunn, J. E., and Rajagopal K. R., Fluids of differential type: critical review and thermodynamic analysis, International Journal of Engineering Science 33, no. 5: 689729:1995.

[54]. Ali, Nasir, A. Zaman, M. Sajid, O. Anwar Bég, M. D. Shamshuddin, and Ali Kadir. "Numerical simulation of time-dependent non-Newtonian nanopharmacodynamic transport phenomena in a tapered overlapping stenosed artery." Nanoscience and Technology: An International Journal 9, no. 3 (2018).

[55]. Rajashekhar, C., Manjunatha G., and Fabian B., Finite element simulation of blood flow through an artery bifurcation: a mathematical model, Malaysian Journal of Mathematical Sciences 11, no. 2: 165-179, 2017.

[56]. Contreras-Silva, Jorge, Vázquez-Hernández M., García-Nocetti D. F., and Acevedo-Contla P., Simulation of an artery with stenosis using the Finite Element Method (FEM), In Global Medical Engineering Physics Exchanges/Pan American Health Care Exchanges (GMEPE/PAHCE), 2017, pp. 1-1. IEEE, 2017.

[57]. Lozovskiy, Alexander, Maxim A. Olshanskii, and Yuri V. Vassilevski., A quasi-Lagrangian finite element method for the Navier-Stokes equations in a time-dependent domain, Computer Methods in Applied Mechanics and Engineering, 333: 55-73, 2018.

[58]. Choudhari, Pranali, and Panse M. S., Finite element modeling and simulation of arteries in the human arm to study the aortic pulse wave propagation, Procedia Computer Science, 93: 721-727, 2016.

[59]. Gupta, Vineeta, Pagey S. S, and Saxena V. P., Numerical analysis of drug diffusion in human dermal region with linear shape function, ISOR J Math., 4, no. 2: 31-6, 2012.

[60]. Khanday, M. A., and Rafiq Aasma, Variational finite element method to study the absorption rate of drug at various compartments through transdermal drug delivery system, Alexandria Journal of Medicine 51, no. 3: 219-223, 2015.

[61]. Sharma J., Srivastava S. K., Four step wavelet Galerkin Method for parabolic and hyperbolic problems, International Journal of Science and Research, 1, 557-562, 2015.

[62]. Shu, Chi-Wang., Discontinuous Galerkin method for time-dependent problems: survey and recent developments, in Recent developments in discontinuous Galerkin finite element methods for partial differential equations, pp. 25-62. Springer, Cham, 2014.

[63]. Bertoglio, Cristóbal, Caiazzo Alfonso, Bazilevs Yuri, Braack Malte, Esmaily Mahdi, Gravemeier Volker, Marsden, Olivier Pironneau Alison L., Vignon-Clementel Irene E., and Wall Wolfgang A., Benchmark problems for numerical treatment of backflow at open 
boundaries, International Journal Numerical Methods Biomedical Engineering 34, no. 2: e2918, 2018.

[64]. Deleuze, Y., Thiriet, M., \& Sheu, T. W., On three-dimensional ALE finite element model for simulating deformed interstitial medium in the presence of a moving needle. Computers \& Fluids, 141, 75-81, 2016.

[65]. Janela, J., Sequeira, A., \& Carapau, F., Numerical simulation of the motion of rigid particles in generalized Newtonian fluids using a hyper-viscosity method. WSEAS Transactions on Mathematics, 5(4), 366, 2006.

[66]. Mahmud K. R., Rhaman M. M. and Al Azad A. K., Numerical simulation and analysis of incompressible Newtonian fluid flows using FreeFem++, Journal of Advanced Research in Fluid Mechanics and Thermal Sciences, 26, no. 1:1-19, 2016.

[67]. Devys, A., Grandmont, C., Grec, B., Maury, B., \& Yakoubi, D., Numerical method for the 2D simulation of the respiration. In ESAIM: Proceedings (Vol. 28, pp. 162-181). EDP Sciences, 2006.

[68]. Sauvage, Emilie, Patient-specific blood flow modelling, Ph. D. Thesis, Université Catholique de Louvain, Belgium, 2014.

[69]. Hecht, Frederic, New development in FreeFEM++, J. Numerical Mathematics, 20, 251-266, 2012.

[70]. Cramer, K.C. and Pai, S., Magnetofluid Dynamics for Engineers and Applied Physicists, MacGraw-Hill, New York, USA, 1973.

[71]. Brix G, Strieth S, Strelczyk D, Dellian M, Griebel J, Eichhorn ME, Andrā W, Bellemann ME, Static magnetic fields affect capillary flow of red blood cells in striated skin muscle, Microcirculation. 15(1):15-26

[72] Fung, Y.C., Biomechanics: Motion, Flow, Stress and Growth, Springer, New York, 1990.

[73] A.P. Yoganathan, EG Cape, HW Sung, FP Williams, A Jimoh, Review of hydrodynamic principles for the cardiologist: applications to the study of blood flow and jets by imaging techniques, Journal of the American College of Cardiology 12 (5), 1344-1353, 1988.

[74] T.F. Zien, Hydrodynamics of bolus flow-An analytical approach to blood flow in capillaries, The Bulletin of Mathematical Biophysics, 31, 681-694, 1969.

[75] Husain, I., C. Langdon, and J. Schwark, Three-dimensional pulsatile non-Newtonian flow in a stenotic vessel. In: Computational Problems in Engineering. Springer, pp. 55-64, 2014. 